\title{
Monitoring the Decoherence of Mesoscopic Quantum Superpositions in a Cavity
}

\author{
Jean-Michel RAIMOND \\ Laboratoire Kastler Brossel \\ Département de Physique de l'Ecole Normale Supérieure \\ 24 rue Lhomond \\ F-75231 Paris Cedex 05, France
}

$\&$

\author{
Serge HaRoche \\ Collège de France \\ 11 place Marcelin Berthelot \\ F-75231 Paris Cedex 05, France
}

\begin{abstract}
Decoherence is an extremely fast and efficient environment-induced process transforming macroscopic quantum superpositions into statistical mixtures. It is an essential step in quantum measurement and a formidable obstacle for a practical use of quantum superpositions (quantum computing for instance). For large objects, decoherence is so fast that its dynamics is unobservable. Mesoscopic fields stored in a high-quality superconducting millimeter-wave cavity, a modern equivalent to Einstein's 'photon box', are ideal tools to reveal the dynamics of the decoherence process. Their interaction with a single circular Rydberg atom prepares them in a quantum superposition of fields, containing a few photons, with different classical phases. The evolution of this 'Schrödinger cat' state can be later probed with a 'quantum mouse', another atom, assessing its coherence. We describe here the experiments performed along these lines at ENS, and stress the deep links between decoherence and complementarity.
\end{abstract}

\section{Introduction}

Quantum state superpositions are ubiquitous in the microscopic world. They are at the heart of any interferometry experiment, be them either the fancy quantum interferences of laser cooled atoms or the usual light interferences observed, for instance, in the Young's double slit device.

Quite obviously, these quantum superpositions do not invade our macroscopic world. At out scale, there is not such a thing that an object following two paths at the same time or, to quote once more Schrödinger's provocative wording, a cat that would be suspended in quantum limbs in a quantum superposition of its 'dead' and 'alive' states. In fact, we observe only a tiny fraction of all possible states of an immense Hilbert space.

Decoherence is the extremely efficient mechanism that confines the weirdness of the quantum world at a microscopic scale and that prevents the quantum monsters from entering our lives $[1,2,3]$. Any quantum system is coupled to an environment. For microscopic systems, made of a single spin, a single atom or a single photon, this coupling results in the usual relaxation mechanism, setting a finite lifetime to the system's energy, for instance.

For a mesoscopic quantum superposition, the environment very rapidly transforms it into a mere statistical mixture, obeying the standard probability laws for exclusive events. The decoherence time scale decreases when a parameter measuring the distance between the states in the superposition increases. For superpositions of very different states, the decoherence time scale is extraordinarily short, making the observation of such quantum superpositions practically impossible (see W. Zurek's and H.D. Zeh's contributions in this Volume).

Observing our classical world is a strong indication of the validity of the decoherence concept. It would, obviously, be much more interesting to study the dynamics of the decoherence, to unveil 


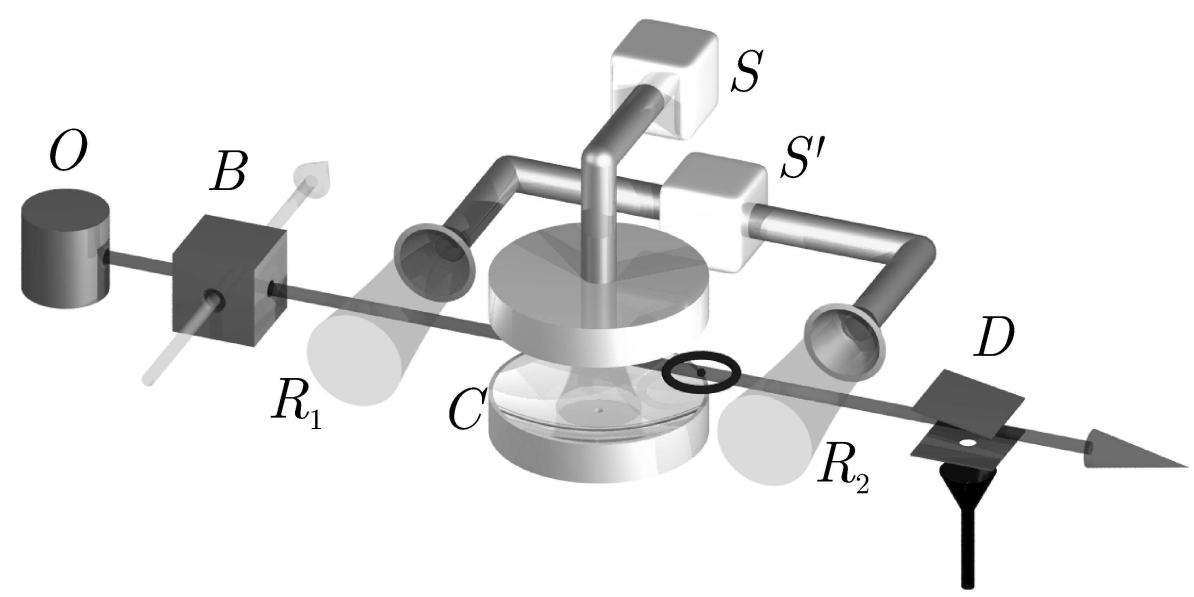

Figure 1: Scheme of the ENS cavity QED set-up.

its inner workings and to put our understanding of this essential phenomenon under close scrutiny.

Testing experimentally decoherence is a challenging task. First, it is so efficient that there is no hope to resolve its dynamics when it comes to macroscopic objects, even much simpler than a cat. One should thus control a single mesoscopic quantum system. Larger than a single atom or a single photon, it should be nevertheless sufficiently isolated from its environment to provide an accessible decoherence time scale. It should first be prepared in a superposition of states at a mesoscopic 'distance' in the system's phase space. Finally, a signal indicating the degree of coherence (of 'quantumness') of the final system's state should be obtained and studied as a function of time, revealing the decoherence dynamics.

Cavity Quantum Electrodynamics [4] and ion trap experiments [5] are ideally suited for these experiments. Both implement a simple quantum system, made up of a single two-level system (a spin-1/2) strongly interacting with a mesoscopic quantum oscillator (a spring). In the cavity QED case, the oscillator is a single mode of a high quality cavity, interacting with a single atom. In the ion trap experiments, the oscillator is the mechanical motion of the ion in the trap, coupled by appropriate laser beams to the ion's internal state. In both cases, the spin/spring interaction can be tailored to cast the oscillator in a mesoscopic quantum state superposition $[6,7]$. The decoherence dynamics, unveiled for the first time in a cavity QED experiment [7], has also been studied in the ion trap context [8].

We shall describe here the cavity QED experiments performed at ENS on 'Schrödinger cat states' of the cavity field and their decoherence [4]. These experiments use circular Rydberg atoms interacting, one at a time, with a superconducting cavity. These cavities are a reasonable modern approximation of the 'photon box' that Bohr and Einstein were using in their heated debates about quantum mechanics. They are able to store microwave fields for long times, up to a millisecond, corresponding to quality factors $Q$ in the $10^{8}$ range. Their mode, equivalent to an harmonic oscillator, is thus pretty well isolated from the outside world.

Circular Rydberg states have a high principal quantum number, around 50 in these experiments, and maximum orbital and magnetic quantum numbers. They have a nearly macroscopic size: the atomic radius is $0.1 \mu \mathrm{m}$, comparable to the size of a living organism, virus or bacteria. They are blessed with remarkable properties [4], being long-lived (radiative lifetime about $30 \mathrm{~ms}$ ) with a huge dipole strongly coupling the transition between two adjacent states to millimeterwaves. The atomic transition frequency can be tuned by Stark effect in a d.c. electric field. Finally, circular atoms can be detected in a selective and sensitive way using field ionization.

Figure 1 presents the ENS experimental scheme. In box $B$, velocity-selected rubidium atoms effusing from oven $O$ are prepared in the circular states $|e\rangle$ or $|g\rangle$, with principal quantum numbers 51 and 50 respectively. The atomic samples are excited at a given time. Their position is thus known at any time during their $20 \mathrm{~cm}$ transit through the apparatus until they are finally detected 
in the field-ionization counter $D$, which discriminates $|e\rangle$ and $|g\rangle$. The atoms are protected from the room-temperature backbody field by a $1 \mathrm{~K}$ cryogenic environment. The atom number in a sample obeys a Poisson statistics, with much less than one atom on the average. When an event is recorded by $D$ (detection efficiency $>80 \%$ ), the probability that it corresponds to more than one atom is negligible. The atomic state can be manipulated before or after the interaction with the cavity $C$ by resonant classical microwave fields produced in the zones $R_{1}$ and $R_{2}$ by the additional source $S^{\prime}$.

The $|e\rangle \rightarrow|g\rangle$ transition, at $51.1 \mathrm{GHz}$, is resonant or nearly resonant with the superconducting Fabry Perot cavity $C$. The photon storage time, $T_{c}$, is of the order of $1 \mathrm{~ms}$. A classical source, $S$, injects classical fields in $C$, with controlled amplitudes and phases. An electric field applied across the mirrors tunes the atomic transition via the Stark effect in or out of resonance with $C$, at well defined times, leading to a precise control of the atom-cavity interaction dynamics.

We shall first, in Section 2, describe the quantum optics theoretical tools necessary to analyze the decoherence experiments. We first briefly recall the field quantization in a single mode, equivalent to a one-dimensional harmonic oscillator. We then describe two important quantum fields, the coherent states and the Schrödinger cat states. The coherent states are the most classical field states, being produced by classical sources such as $S$. They are also the most stable with respect to cavity relaxation, the 'pointer states' for the field. The cat states are quantum superpositions of different coherent states and present remarkable quantum features. Finally, we describe cavity mode relaxation, either in the Master equation or in the Monte Carlo trajectories approaches, which shed complementary lights on this important process.

Section 3 is devoted to the production an detection of Schrödinger cat states in the cavity. We describe first the atom-cavity interaction in terms of the 'dressed sates'. We show that the interaction with a single, non-resonant atom transforms a coherent state into a cat, whose decoherence is then theoretically analyzed. We explain how a probe atom, a 'quantum mouse' can be used to assess the decoherence of the cavity cat.

Finally, Section 4 is devoted to the perspectives opened for these decoherence studies: creation of very large cats by resonant atom-field interaction, direct measurement of the cat's Wigner function, providing a detailed insight into the decoherence mechanisms and creation of non-local cat states, merging the Einstein-Podolsky-Rosen [9] non-locality and decoherence.

\section{A quantum field at the boundary of quantum and classical worlds}

\subsection{A quantum cavity mode}

A single field mode is equivalent to a one-dimensional harmonic oscillator. The non-degenerate energy eigenstates are the Fock or 'photon number states' $\{|n\rangle\} n>0$, whose energy is $\hbar \omega_{c}\left(n+\frac{1}{2}\right)$, where $\omega_{c}$ is the cavity mode angular frequency. The ground state is the vacuum $|0\rangle$. The Fock states are an orthogonal set:

$$
\langle n \mid p\rangle=\delta_{n p} .
$$

The photon annihilation and creation operators $a$ and $a^{\dagger}$ connect the Fock states:

$$
a|n\rangle=\sqrt{n}|n-1\rangle ; \quad a^{\dagger}|n\rangle=\sqrt{n+1}|n+1\rangle .
$$

The action of $a$ on $|0\rangle$ gives a null vector (it is not possible to annihilate a photon in vacuum). All Fock states can be generated from the vacuum by repeated applications of the photon creation operator: $|n\rangle=a^{\dagger n}|0\rangle \sqrt{n !}$. These operators obey a bosonic commutation rule: $\left[a, a^{\dagger}\right]=1$.

The cavity Hamiltonian is $H_{c}=\hbar \omega_{c}\left(a^{\dagger} a+1 / 2\right)$. Since we are dealing with a single-mode situation, we can redefine the energy origin to get rid of the non-vanishing vacuum state energy. We can thus also use the simpler Hamiltonian:

$$
H_{c}^{\prime}=\hbar \omega_{c} a^{\dagger} a
$$

The cavity mode electric field operator at position $\mathbf{r}$ writes:

$$
\mathbf{E}_{c}=i \mathcal{E}_{0}\left[\mathbf{f}(\mathbf{r}) a-\mathbf{f}^{*}(\mathbf{r}) a^{\dagger}\right],
$$




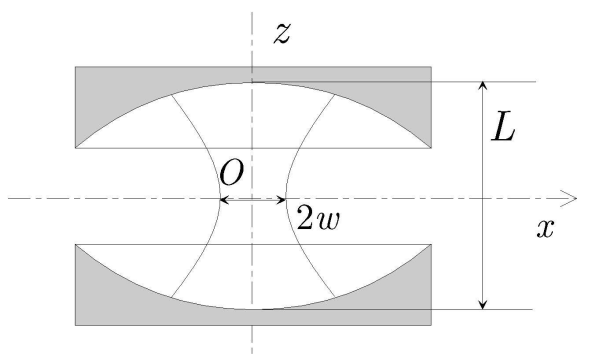

Figure 2: Scheme of a microwave Fabry-Perot cavity. The two spherical mirrors separated by $L$ sustain a Gaussian mode with waist $w$. The cavity axis normal to the mirrors is $O z$. The sinusoidal standing wave pattern (with field nodes and antinodes along $O z$ ) is not represented. Atoms propagate along $O x$ axis.

where $\mathcal{E}_{0}$ is a normalization factor. The dimensionless vector function $\mathbf{f}(\mathbf{r})=\boldsymbol{\epsilon}_{c} f(\mathbf{r})$ describes the spatial structure of the field mode (relative field amplitude $f$ and polarization $\boldsymbol{\epsilon}_{c}$ ). At the point where the field mode amplitude is maximum, which we also take as the origin, $f=1$.

The normalization factor is obtained by equating the Fock states energies with the integral over space of the expectation value of the electromagnetic energy density $\varepsilon_{0}\left|\mathbf{E}_{c}\right|^{2}$ :

$$
\mathcal{E}_{0}=\sqrt{\frac{\hbar \omega_{c}}{2 \varepsilon_{0} \mathcal{V}}}
$$

where we define the cavity effective volume $\mathcal{V}$ by:

$$
\mathcal{V}=\int|\mathbf{f}(\mathbf{r})|^{2} d V
$$

As a specific example, consider the case of the Fabry-Perot cavity briefly described in Section 1. It is made of two spherical mirrors facing each other (Figure 2). The mode is then a standing wave with a Gaussian transverse profile and a sinusoidal field variation in the longitudinal direction normal to the mirrors, separated by the distance $L$. The waist $w$ characterizes the minimum width of the Gaussian. The mode volume is then $V=\pi L w^{2} / 4$. For the specific parameters of the experiment $(L=2.7 \mathrm{~cm} ; w=6 \mathrm{~mm})$ we have $V=0.7 \mathrm{~cm}^{3}$. The field per photon is then:

$$
\mathcal{E}_{0}=1.510^{-3} \mathrm{~V} / \mathrm{cm},
$$

a rather large value in S.I. units for a quantum field.

The field quadrature operators correspond to a mechanical oscillator's position and momentum:

$$
X=\frac{a+a^{\dagger}}{2} \quad ; \quad P=\frac{a-a^{\dagger}}{2 i}=\frac{e^{-i \pi / 2} a+e^{i \pi / 2} a^{\dagger}}{2} .
$$

More generally, phase quadratures are linear combinations of $a$ and $a^{\dagger}$ :

$$
X_{\varphi}=\frac{e^{-i \varphi} a+e^{i \varphi} a^{\dagger}}{2} ; X_{\varphi+\pi / 2}=\frac{e^{-i \varphi} a-e^{i \varphi} a^{\dagger}}{2 i} .
$$

They satisfy the commutation rules $\left[X_{\varphi}, X_{\varphi+\pi / 2}\right]=i / 2$, which correspond to the uncertainty relations $\Delta X_{\varphi} \Delta X_{\varphi+\pi / 2} \geq 1 / 4$, where $\Delta X_{\varphi}$ and $\Delta X_{\varphi+\pi / 2}$ are conjugate phase quadrature fluctuations.

The eigenstate of the quadrature $X_{\varphi}$ corresponding to the real and continuous eigenvalue $x$ is a non-normalizable state (infinite energy), which obeys the orthogonality and closure relationships:

$$
{ }_{\varphi}\left\langle x \mid x^{\prime}\right\rangle_{\varphi}=\delta\left(x-x^{\prime}\right) ; \quad \int|x\rangle_{\varphi \varphi}\langle x| d x=\mathbb{1}
$$



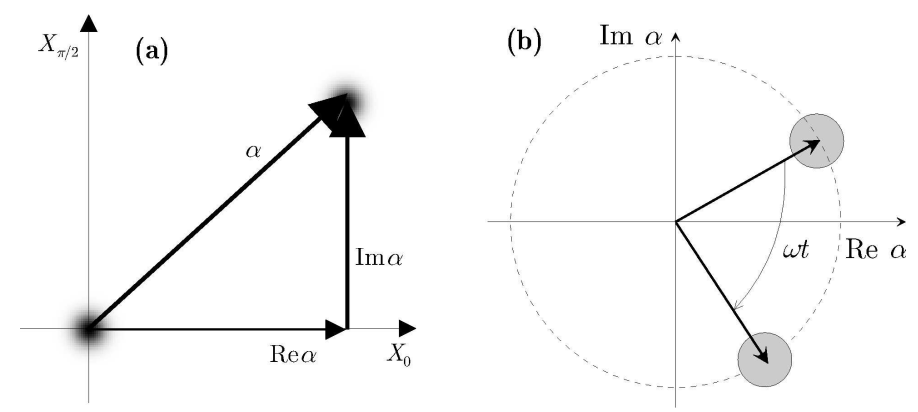

Figure 3: Coherent state. (a) Pictorial representation of the action of the displacement operator on the vacuum state. The displacement by a complex amplitude $\alpha$ amounts to a displacement by $\operatorname{Re} \alpha$ along the $X_{0}$ quadrature axis, followed by a displacement by $\operatorname{Im} \alpha$ along the $X_{\pi / 2}$ quadrature. (b) Time evolution of a coherent state.

the transformation from the $|x\rangle_{\varphi}$ basis to the conjugate basis $|x\rangle_{\varphi+\pi / 2}$ being a Fourier transform:

$$
|x\rangle_{\varphi+\pi / 2}=\int d y|y\rangle_{\varphi \varphi}\langle y \mid x\rangle_{\varphi+\pi / 2}=\frac{1}{\sqrt{\pi}} \int d y e^{2 i x y}|y\rangle_{\varphi} .
$$

Note that two conjugate field quadratures provide coordinates for the quantum field phase space, equivalent to the Fresnel plane for classical fields.

The expectation value $\left\langle n\left|X_{\varphi}\right| n\right\rangle$ of any phase quadrature in a Fock state is zero. There is thus no preferred phase neither in the vacuum nor in any Fock state, a feature which shows that these quantum states are quite different from classical fields. For the vacuum, the quadrature fluctuations are isotropic and correspond to the minimum value compatible with Heisenberg uncertainty relations: $\Delta X_{\varphi}^{(0)}=\sqrt{\left\langle 0\left|X_{\varphi}^{2}\right| 0\right\rangle}=1 / 2$. The probability distribution $P^{(0)}(x)$ of the field quadrature is then a Gaussian:

$$
P^{(0)}(x)=\left.\left.\right|_{\varphi}\langle x \mid 0\rangle\right|^{2}=\left(\frac{2}{\pi}\right)^{1 / 2} e^{-2 x^{2}} .
$$

In summary, the vacuum field in each mode has isotropic Gaussian fluctuations around zero field.

\subsection{Coherent states}

To describe situations in which the phase of the field is relevant, it is convenient to expand the field on the basis of the coherent states $[10,11]$, which are more physical than Fock or quadrature states and are experimentally more accessible.

A coherent state of a single field mode is defined as resulting from the translation of the vacuum field in phase space. This translation is represented, in its most general form, by the unitary displacement operator:

$$
D(\alpha)=e^{\alpha a^{\dagger}-\alpha^{*} a},
$$

where $\alpha=|\alpha| \exp (i \phi)$ is a $C$-number whose real and imaginary parts are the projections along the $X_{0}$ and $X_{\pi / 2}$ directions respectively of the translation vector. The translated vacuum state is the coherent state $|\alpha\rangle$ :

$$
|\alpha\rangle=D(\alpha)|0\rangle \text {. }
$$

The translated 'packet', whose evolution is determined in the Schrödinger picture by the free field Hamiltonian [Eq. (3)], subsequently rotates at frequency $\omega_{c}$ in phase space, without deformation: $|\alpha(t)\rangle=\left|\alpha e^{-i \omega_{c} t}\right\rangle$. This corresponds to the best possible approximation of a classical free oscillator motion. Fig. 3(a,b) shows how the vacuum state is transformed by translation into a coherent state and how this state freely evolves in phase space. In Fig. 3(b), the coherent states are pictorially shown as uncertainty circles of radius unity at the tip of the classical amplitude, a representation that we shall repeatedly use in the following. 


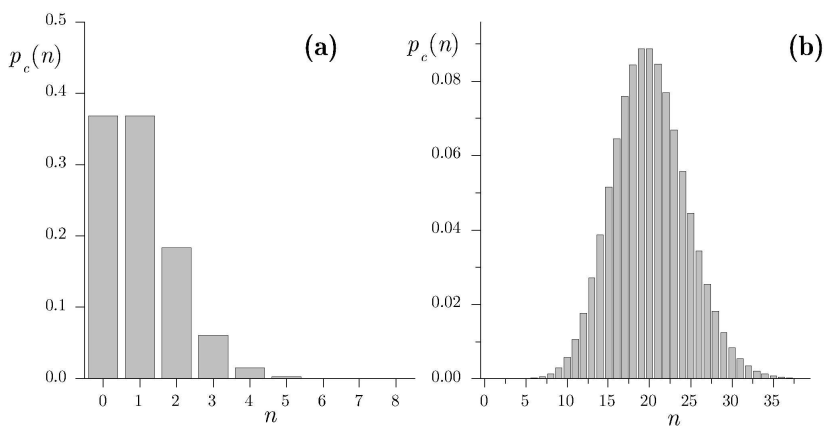

Figure 4: Photon number statistical distributions. (a) Coherent field with $\bar{n}=1$ photons on the average. (b) Coherent field with $\bar{n}=20$.

In order to make the translation operation more explicit, it is convenient to split the exponential in Eq.(13) in two, separating the contributions of the real and imaginary parts of $\alpha$. We make use of the Glauber relation $[12] e^{A+B}=e^{A} e^{B} e^{-[A, B] / 2}$ (valid if $[A, B]$ commutes with $A$ and $B)$ and we get:

$$
D(\alpha)=e^{-i \alpha_{1} \alpha_{2}} e^{2 i \alpha_{2} X_{0}} e^{-2 i \alpha_{1} X_{\pi / 2}}
$$

Using a mechanical oscillator analogy, the displacement $D(\alpha)$ can thus be viewed as a translation along space by an amount $\alpha_{1}=\operatorname{Re}(\alpha)$, followed by a 'momentum kick' of magnitude $\alpha_{2}=\operatorname{Im}(\alpha)$.

Let us compute the probability amplitude for finding the value $x$ when measuring the quadrature operator $X_{0}$ on a field in state $|\alpha\rangle$. A straightforward calculation using Eqs.(14) and (15) yields:

$$
\langle x \mid \alpha\rangle=\left(\frac{2}{\pi}\right)^{1 / 4} e^{-i \alpha_{1} \alpha_{2}} e^{2 i \alpha_{2} x} e^{-\left(x-\alpha_{1}\right)^{2}},
$$

a Gaussian wave packet centered in $\alpha_{1}$, with a phase modulation at frequency $\alpha_{2}$ describing the momentum kick. The probability for finding the value $x$ for the quadrature is thus:

$$
P(x)=\left(\frac{2}{\pi}\right)^{1 / 2} \exp \left[-2\left(x-\alpha_{1}\right)^{2}\right]
$$

a translated ground state distribution.

An alternative and useful expression of the displacement operator is obtained by using again the Glauber relation, separating this time the $a$ and $a^{\dagger}$ terms:

$$
D(\alpha)=e^{\alpha a^{\dagger}-\alpha^{*} a}=\exp \left(-\frac{|\alpha|^{2}}{2}\right) e^{\alpha a^{\dagger}} e^{-\alpha^{*} a} .
$$

This form corresponds to the 'normal ordering' in quantum optics. If we expand the exponential of operators in series, all the $a^{n}$ terms are on the right and the $a^{\dagger n}$ terms on the left. The action of the $\exp \left(-\alpha^{*} a\right)$ operator on the right leaves the vacuum unchanged, since only the zero-order term in the expansion yields a non-zero result. Combining Eqs.(14)and (18), we get:

$$
|\alpha\rangle=\sum_{n} c_{n}(\alpha)|n\rangle \quad \text { with } \quad c_{n}(\alpha)=\exp \left(-\frac{|\alpha|^{2}}{2}\right) \frac{\alpha^{n}}{\sqrt{n !}} .
$$

The distribution of photon numbers in a coherent state obeys a Poisson statistics. Fig. 4 shows this distribution for $\alpha=1$ and $\alpha=\sqrt{20}$. The average photon number $\bar{n}$ and photon number variances $\Delta n$ are:

$$
\bar{n}=|\alpha|^{2} \quad ; \quad \frac{\Delta n}{\bar{n}}=\frac{1}{|\alpha|}=\frac{1}{\sqrt{\bar{n}}} .
$$




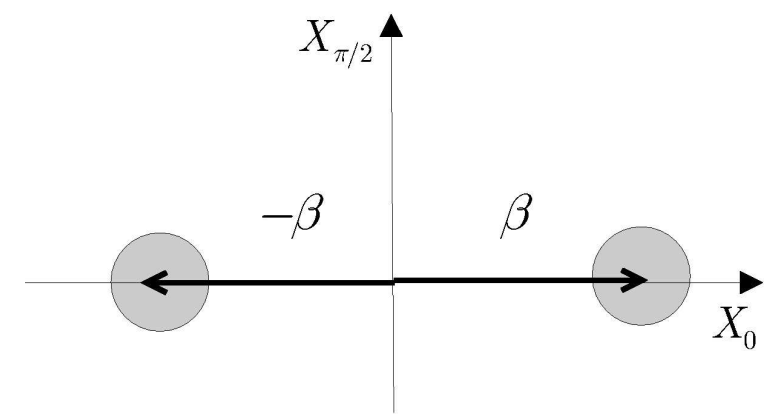

Figure 5: Pictorial representation of a $\pi$-phase cat in phase space.

The relative fluctuation of the photon number is thus inversely proportional to the square root of its average. For large fields, this fluctuation becomes negligible (classical limit).

Coherent states are eigenstates of the photon annihilation operator $a$. This essential property is easily derived from Eqs.(2) and (19):

$$
a|\alpha\rangle=\alpha|\alpha\rangle \quad \text { and } \quad\langle\alpha| a^{\dagger}=\langle\alpha| \alpha^{*} .
$$

It is also useful to recall the expression of the scalar product of two coherent states:

$$
\langle\alpha \mid \beta\rangle=e^{-|\alpha|^{2} / 2-|\beta|^{2} / 2+\alpha^{*} \beta} \quad ; \quad|\langle\alpha \mid \beta\rangle|^{2}=e^{-|\alpha-\beta|^{2}},
$$

which shows that the overlap of two such states decreases exponentially with their 'distance' in phase space. Although they are never strictly orthogonal, they become practically so when the distance of their centers is much larger than 1 , the radius of the uncertainty circle.

The coherent states constitute a complete set of states in the mode's Hilbert space:

$$
\frac{1}{\pi} \int d \alpha_{1} d \alpha_{2}|\alpha\rangle\langle\alpha|=\mathbb{1}
$$

Note however that the non-orthogonal coherent state basis is over-complete. The expansion of a state over it is not unique.

Coherent states are easily produced experimentally, by the classical microwave source $S$ weakly coupled to the cavity mode (see figure 1). The evolution operator for the mode state under the coupling with a classical current is the displacement operator, transforming the initial vacuum in a coherent state whose amplitude and phase are under experimenter's control.

As a classical field, a coherent state is defined by a complex amplitude, evolving in the Fresnel plane. The non-vanishing quantum fluctuations of the field quadratures is represented by the uncertainty circle in Figure 3. For very small fields (about one photon on the average) the amplitude is comparable to the uncertainties and quantum fluctuations play an important role. For very large amplitudes, quantum fluctuations are negligible and the coherent state can be viewed as a classical object, with well defined phase and amplitude. Coherent states stored in a cavity thus span the quantum to classical transition, with the mere adjustment of the source controls.

\subsection{Schrödinger cat states}

We give here a special attention to superpositions of two quasi-orthogonal coherent states, represented in the Fresnel plane by two non-overlapping circles. These states are prototypes of Schrödinger cats $[13,14]$. We will see later how they can be prepared and used to study the dynamics of decoherence. We enumerate first some of their properties. As a simple example, we consider a linear superposition with equal weights of two coherent states with opposite phases (see Fig. 5). This superposition, called a $\pi$-phase cat in the following, writes:

$$
\left|\Psi_{\text {cat }}^{\mathrm{even}}\right\rangle=\frac{|\beta\rangle+|-\beta\rangle}{\sqrt{2\left(1+e^{-2|\beta|^{2}}\right)}} \approx(1 / \sqrt{2})(|\beta\rangle+|-\beta\rangle),
$$


where $\beta$ is the amplitude of the field (taken real). The superscript 'even' will be explained below. The denominator in the first r.h.s. term is a normalization factor, taking into account the overlap of $|\beta\rangle$ and $|-\beta\rangle$. If $|\beta| \gg 1$, this overlap is negligible and the cat state is expressed by the simpler form given by the second r.h.s. term in Eq.(24).

The coherence between the two states is an essential feature which distinguishes it from a mere statistical mixture. This is made clear by expressing the field density operator:

$$
\rho_{\text {cat }} \approx \frac{1}{2}(|\beta\rangle\langle\beta|+|-\beta\rangle\langle-\beta|+| \beta\rangle\langle-\beta|+|-\beta\rangle\langle\beta|) .
$$

The cat coherence is described by the off-diagonal part of this density operator [last two terms in the r.h.s. of Eq.(25)].

This coherence is displayed by analyzing the field quadrature distribution. Suppose first that we measure $X_{0}$. Its probability distribution is the sum of two Gaussians, centered at $\pm \beta$ :

$$
P_{0}^{(\text {cat })}(x) \approx \frac{1}{\sqrt{2 \pi}}\left(e^{-2(x-\beta)^{2}}+e^{-2(x+\beta)^{2}}\right) .
$$

The probability amplitudes for measuring $x$ in state $|+\beta\rangle$ and $|-\beta\rangle$ do not appreciably overlap and thus cannot interfere: the resulting distribution is simply the sum of those corresponding to the state components. The state coherence is not apparent here. If we measure instead $X_{\pi / 2}$, the probability distribution is:

$$
\left.P_{\pi / 2}^{(\mathrm{cat})}(x) \approx \frac{1}{2}\right|_{\pi / 2}\langle x \mid \beta\rangle+\left._{\pi / 2}\langle x \mid-\beta\rangle\right|^{2},
$$

where the index $\pi / 2$ in ${ }_{\pi / 2}\langle x|$ indicates an eigenstate of $X_{\pi / 2}$. The probability $P_{\pi / 2}^{(\text {cat })}(x)$ is the square of the sum of two amplitudes which are both non-zero. These amplitudes are easy to compute. The scalar product of $|x\rangle_{\pi / 2}$ with $|\beta\rangle$ is equal to the product of $|x\rangle_{0}$ with $|-i \beta\rangle$, as a mere rotation in phase space indicates. Using Eq.(16), we get:

$$
P_{\pi / 2}^{(\mathrm{cat})} \approx\left(\frac{2}{\pi}\right)^{1 / 2} e^{-2 x^{2}}(1+\cos 4 \beta x) .
$$

The probability distribution is a Gaussian centered at $x=0$, modulated by an interference term with fringes having a period $1 / 4 \beta$, inversely proportional to the 'cat size' $\beta$. This interference is a signature of the coherence of the superposition.

The distribution of any phase quadrature $X_{\varphi}$ can be obtained in the same way. The interference term exists only when $\varphi$ is close to $\pi / 2$. A graphical representation is very convenient to understand why it is so (Fig. 6). For a coherent state, a field quadrature takes non-zero values in an interval corresponding to the projection of the state uncertainty circle on the direction of the quadrature. For a Schrödinger cat state, there are two such intervals, corresponding to the two state components. If $\beta \gg 1$ and $\varphi=0$ [Fig. 6(a)], the two intervals are non-overlapping and there is no interference. For a $\varphi$ value between 0 and $\pi / 2$ [Fig. 6(b)], the two intervals are closer than for $\varphi=0$, resulting in two still non-overlapping gaussians without interference. It is only when $\varphi$ gets very close to $\pi / 2$ that the two projected intervals overlap along the direction of the quadrature, leading to a large interference term [Fig. 6(c)].

Another aspect of the cat states coherence is revealed by considering their photon number distribution. The state given by Eq.(24) develops only along even number states, since the probability for finding $n$ photons in it is proportional to $1+(-1)^{n}$, justifying the superscript 'even' in its name. Similarly the cat state:

$$
\left|\psi^{\text {odd }}\right\rangle=(1 / \sqrt{2})[|\beta\rangle-|-\beta\rangle]
$$

develops only along the odd photon numbers. We call it an 'odd phase cat'. The periodicity of the photon number is related to the coherence of the state, since a statistical mixture of $|\beta\rangle$ and $|-\beta\rangle$ contains all photon numbers. The modulated photon number distribution is a signature of the even and odd cats coherence, as is the existence of dark fringes in their $X_{\pi / 2}$ quadrature. 


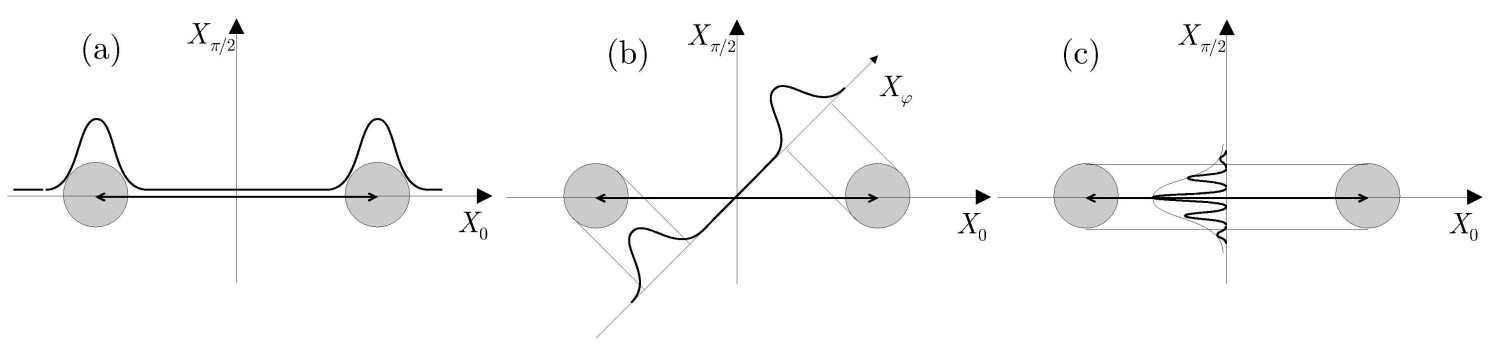

Figure 6: Quadratures of an even Schrödinger cat. (a) The $X_{0}$ quadrature exhibits well separated Gaussian peaks corresponding to the cat's components. (b) For $X_{\pi / 4}$, the Gaussian peaks distance is reduced. (c) For $X_{\pi / 2}$ the two peaks merge and fringes show up.

It is hence convenient to introduce the photon number parity operator $\mathcal{P}$ [12] which admits as eigenstates all the superpositions of even photon numbers with the eigenvalue +1 and all the superpositions of odd photon number states with the eigenvalue 1:

$$
\mathcal{P}=e^{i \pi a^{\dagger} a} .
$$

The odd and even phase cats $|\beta\rangle \pm|-\beta\rangle$ are eigenstates of $\mathcal{P}$ with the +1 and -1 eigenvalues. From Eq.(21), the action of the annihilation operator on an even (odd) phase cat results in the switching of the cat parity:

$$
a[|\beta\rangle \pm|-\beta\rangle]=\beta[|\beta\rangle \mp|-\beta\rangle] .
$$

Let us remark finally:

$$
\mathcal{P}|x\rangle_{\varphi}=|-x\rangle_{\varphi}
$$

\subsection{A pictorial representation of field states}

We used up to now qualitative phase-space representations. These pictures are made fully quantitative by associating unambiguously to the field state, pure or statistical mixture, two functions, $Q$ and $W$, taking real values in phase space [15] and extending in the quantum realm the probability distributions over phase space, which are pivotal in statistical mechanics. They can be plotted and give a vivid description of the state. The knowledge of any one of them is sufficient to reconstruct the field density operator and thus the result probability distribution for any measurement.

\subsubsection{The $Q$ function}

The field is described by the density operator $\rho$. Its $Q$ function at the point in phase space defined by the complex amplitude $\alpha$ is:

$$
Q(\alpha)=\frac{1}{\pi}\langle\alpha|\rho| \alpha\rangle=\frac{1}{\pi} \operatorname{Tr}[\rho|\alpha\rangle\langle\alpha|] .
$$

For a pure state, $Q$ is the square of the overlap with a coherent state whose amplitude spans the Fresnel plane. It is a real, positive and normalized quantity: the integral of $Q$ over the whole phase space is equal to 1 . An alternative definition of $Q$ is, using Eq.(14):

$$
Q(\alpha)=\frac{1}{\pi} \operatorname{Tr}[|0\rangle\langle 0| D(-\alpha) \rho D(\alpha)]
$$

Thus, $Q(\alpha)$ is the expectation value of the projector on the vacuum, in the state of the field translated by $-\alpha$, leading, as shown below, to a simple experimental determination of $Q$ [16].

It results directly from Eqs.(33) and (22) that $Q(\alpha)$ is a Gaussian centered in $\beta$ for the coherent state $|\beta\rangle$ [Fig. 7(a)]. The $Q$ function of a Schrödinger cat defined by Eq.(24) is essentially the superposition of two gaussians, centered at $\pm \beta$ [Fig. 7(b)]. There is a small additional interference term taking non-zero values between these two gaussians, but it is of the order of the scalar product 


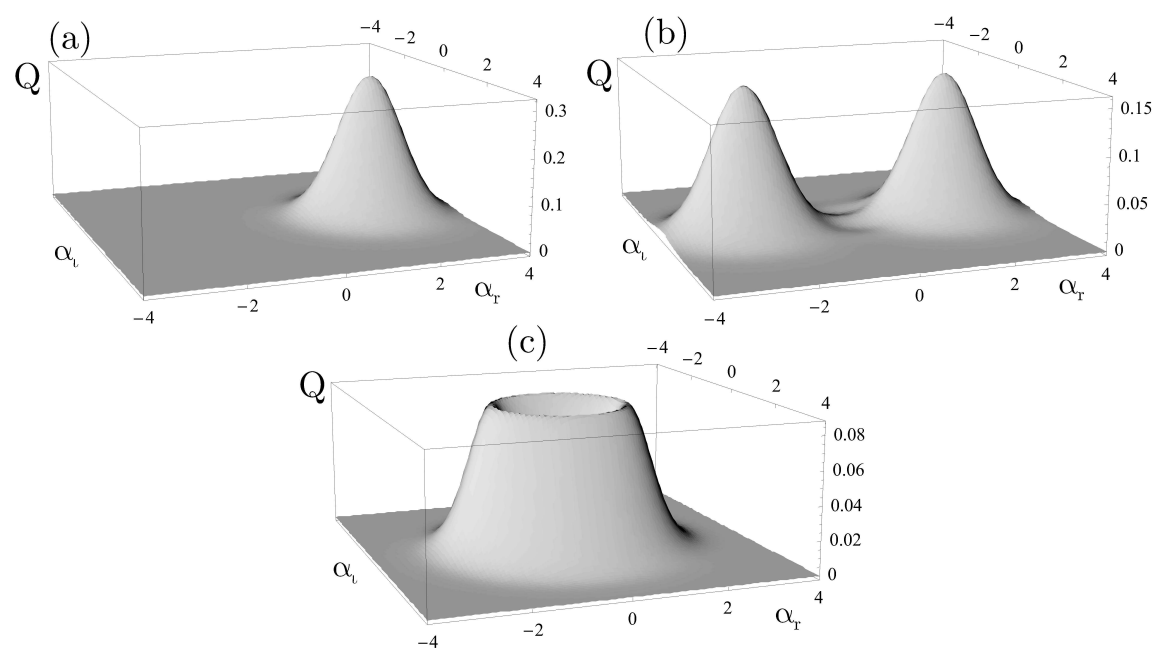

Figure 7: Plots of $Q$ functions. (a) 5 photons coherent state (real amplitude). (b) Schrödinger even cat state, superposition of two 5 photon coherent states with opposite phases. Note the weak interference pattern near the origin. (c) Two-photon Fock state.

of the two cat components, vanishingly small as soon as they are separated. The $Q$ function is thus not appropriate to describe the coherence of a cat. Fig. 7(c) shows the $Q$ function of a $n=2$ Fock state, a circular rim around the origin as intuitively expected.

\subsubsection{The Wigner function}

The Wigner distribution $W(\alpha)=W(x+i p)[17]$ is defined by:

$$
W(x, p)=\frac{1}{\pi} \int d x^{\prime} e^{-2 i x^{\prime} p}\left\langle x+\frac{x^{\prime}}{2}|\rho| x-\frac{x^{\prime}}{2}\right\rangle .
$$

It is the Fourier transform of an off-diagonal matrix element of $\rho$ in a quadrature representation. The $W$ function is real and normalized. Contrary to $Q$, it can take negative values in domains of phase space, making it clear that it is not a probability distribution. Negative values are, as shown below, a signature of non-classical states.

The $W$ function of coherent states are again Gaussian functions centered at the amplitude of the state, but their width is $\sqrt{2}$ times smaller than the one of $Q$. The $W$ function of a $\pi$-phase cat is made up of two Gaussian peaks and a large interference pattern between these peaks with an alternance of positive and negative ridges [Fig.8(a)]. This pattern is a signature of the cat's coherence, lacking in the $W$ function of a statistical mixture. The $W$ function is thus much better adapted than $Q$ for the study of a cat's coherence. Fig. 8(b) and (c) present the $W$ function of the $n=1$ and $n=2$ Fock states which also exhibit negative parts.

By inverse Fourier transform of Eq.(35), the matrix elements of the field density operator are:

$$
\left\langle x+\frac{x^{\prime}}{2}|\rho| x-\frac{x^{\prime}}{2}\right\rangle=\int d p e^{2 i x^{\prime} p} W(x, p) .
$$

The field density operator is thus fully determined by $W$. In particular, the probability density of the quadrature $X_{0}$, is:

$$
\langle x|\rho| x\rangle=\int d p W(x, p) .
$$

The probability that $X_{0}$ takes a given value $x$ is obtained by integrating $W$ for this $x$ value, along all values of $p$. This property holds for any set of orthogonal quadratures. Schrödinger cats, for instance, have quadratures values occurring with 0 probability (see Figure 6), a signature of 

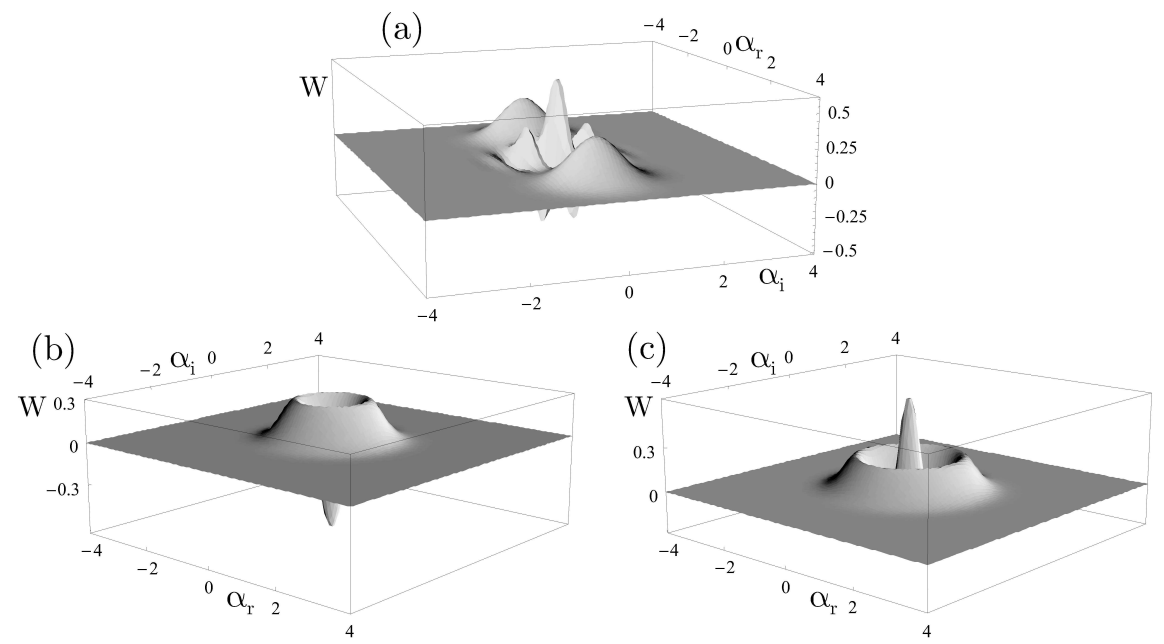

Figure 8: Plot of $W$ functions. (a) Schrödinger cat, superposition of two 5-photon coherent states with opposite phases. (b) One-photon Fock state. (c) Two-photon Fock state.

their non-classical nature. The integral of $W$ along the orthogonal quadrature vanishes, which is possible only if $W$ presents alternations of positive and negative values. We thus understand that negativities of $W$ are related to non-classicality.

We conclude by giving an alternative expression of $W$ ([18] and L. Davidovich, private communication). Using:

$$
\left|x-\frac{x^{\prime}}{2}\right\rangle=e^{-i\left(x-x^{\prime}\right) p} D(x+i p)\left|-\frac{x^{\prime}}{2}\right\rangle
$$

and

$$
\left\langle x+\frac{x^{\prime}}{2}\right|=\left\langle\frac{x^{\prime}}{2}\right| D(-x-i p) e^{i\left(x+x^{\prime}\right) p}
$$

which follow directly from the definition of $D(\alpha)$ and replacing $\left|x \pm x^{\prime} / 2\right\rangle$ in Eq.(36) by the expressions given by Eqs.(38) and (39), noting finally that $\mathcal{P}\left|x^{\prime} / 2\right\rangle=\left|-x^{\prime} / 2\right\rangle$ [see Eq. (32)], we get:

$$
W(x, p)=\frac{2}{\pi} \operatorname{Tr}[D(-\alpha) \rho D(\alpha) \mathcal{P}] .
$$

The Wigner distribution at $\alpha$ is the expectation value in the state translated by $-\alpha$ of the field parity operator. This property leads to a simple experimental determination of $W$ described in Section 4.2.

\subsection{Field mode relaxation}

\subsubsection{Master equation}

Decoherence is the result of a dissemination of information about a system via its entanglement with an environment $E$. For all practical purposes, this information is buried in $E$ on which no measurement can be performed in practice. The system density operator $\rho$ is obtained by tracing over $E$ the system/environment entangled state.

The system relaxation is then described by a master equation, differential equation for $\rho$ alone. This equation can be derived from a simple environment model [12], a bath of harmonic oscillators, for instance, spanning a wide range of frequencies around the system's eigenfrequencies, in thermal equilibrium at a finite temperature $T$. Quite remarkably, the final form of the master equation is model-independent. 


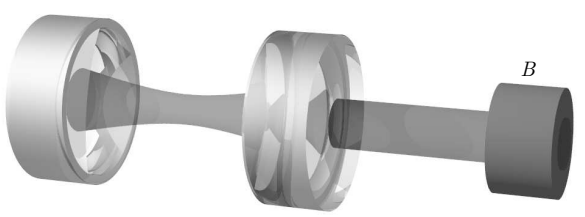

Figure 9: An environment $B$ detecting the photon lost by the cavity field.

For the sake of simplicity, we consider only the zero temperature case. The master equation then writes in the standard Lindblad form:

$$
\frac{d \rho}{d t}=-\frac{i}{\hbar}\left[H_{c}^{\prime}, \rho\right]+\frac{\kappa}{2}\left[2 a \rho a^{\dagger}-a^{\dagger} a \rho-\rho a^{\dagger} a\right],
$$

where $\kappa=1 / T_{c}=\omega_{c} / Q$ the damping rate of the cavity mode energy. The first term in the r.h.s. describes the Hamiltonian evolution. The second describes the effect of photon escape into $E$.

Remarkably, this equation depends only upon the classical energy damping time $T_{c}=1 / \kappa$, which can be measured with macroscopic fields. At finite temperature, additional terms, proportional to $n_{t h}$, the mean number of thermal photons per mode, describe the creation of thermalexcitations in the mode.

It is easy to derive from Eq.(41) the evolution of the photon number distribution $p(n)=$ $\rho_{n n}=\langle n|\rho| n\rangle:$

$$
\frac{d p(n)}{d t}=\kappa(n+1) p(n+1)-\kappa n p(n) .
$$

This equation describes a cascade in the Fock states ladder. The lifetime of state $|n\rangle$ is thus of the order of $T_{c} / n$, decreasing when the number of photon increases. We show below that the lifetime of a coherent state is independent upon its amplitude. That non-classical Fock states are more fragile than semi-classical coherent ones is a first insight into decoherence. We defer to the next Section the application of the master equation to a cat state.

\subsubsection{Monte Carlo trajectories}

The master equation does not provide a detailed insight into the mechanisms of relaxation. The Monte Carlo wavefunction approach is more convenient for computational purposes and also more insightful. We give here the recipes for the Monte Carlo method in the cavity case and discuss briefly its physical contents. A more detailed account can be found in [19, 20].

The master equation is obtained by tracing over all possible results of a virtual measurement performed over the environment. Let us imagine instead that this measurement is explicitly made and its results recorded. What happens then? For the sake of definiteness, the cavity will be owned by a first operator, called Alice, and Bob has a complete control over the environment.

Bob is to monitor relaxation events, corresponding to the loss of a photon by the mode. He could, for instance (see figure 9) couple the cavity with a detector, registering a click whenever a photon escapes.

Let us consider a short time interval $\tau$. At the beginning, the cavity is in a pure state $\left|\phi^{(A)}\right\rangle$ and Bob's detector in a neutral state $\left|0^{(B)}\right\rangle$. During the time interval $\tau$, the system evolves according to the infinitesimal unitary transformation:

$$
\left|\phi^{(A)}\right\rangle \otimes\left|0^{(B)}\right\rangle \rightarrow\left[1-\frac{1}{2} \kappa \tau\left(a^{\dagger} a\right)\right]\left|\phi^{(A)}\right\rangle \otimes\left|0^{(B)}\right\rangle+\sqrt{\kappa \tau} a\left|\phi^{(A)}\right\rangle \otimes\left|1^{(B)}\right\rangle,
$$

in which $\left|1^{(B)}\right\rangle$, orthogonal to $\left|0^{(B)}\right\rangle$, is the detector's state after photon detection. The time interval $\tau$ being very short, the probability for loosing two photons is negligible. The cavity/environment state is entangled, an essential feature of decoherence. 
At the end of this time interval, Bob performs a measurement on its detector, in the $\left\{\left|0^{(B)}\right\rangle\right.$, $\left.\left|1^{(B)}\right\rangle\right\}$ basis. The probabilities $p_{1}$ and $p_{0}$ for finding the environment (equivalent to a single qubit in this simple situation) in $\left|1^{(B)}\right\rangle$ or $\left|0^{(B)}\right\rangle$ are:

$$
p_{1}=1-p_{0}=\kappa \tau\left\langle\phi^{(A)}\left|a^{\dagger} a\right| \phi^{(A)}\right\rangle .
$$

Depending on the outcome of the measurement, the cavity state is projected in one of the states defined as:

$$
\left|\phi_{1}^{(A)}\right\rangle=\frac{a\left|\phi^{(A)}\right\rangle}{\sqrt{p_{1}}},
$$

for a photon click and:

$$
\left|\phi_{0}^{(A)}\right\rangle=\frac{\left[1-\frac{1}{2} \kappa \tau a^{\dagger} a\right]\left|\phi^{(A)}\right\rangle}{\sqrt{p_{0}}} .
$$

when no click is recorded. Eq.(45) justifies the 'jump operator' name coined for $\sqrt{\kappa} a$, since it describes the discontinuous change of the mode state when $B$ is found in $\left|1^{(B)}\right\rangle$. In the event that $B$ does not change, Eq.(46) shows that the oscillator state is also modified. It evolves under the effect of an infinitesimal non-unitary transformation produced by the anti-hermitian pseudoHamiltonian $i \hbar \kappa a^{\dagger} a$. This non-unitary transformation can be described as a renormalization of the oscillator frequency by the addition of an imaginary term in its frequency $(\omega \rightarrow \omega-i \kappa / 2)$. It does not conserve the norm of the state, hence the necessity to normalize it by the $\left(p_{0}\right)^{-1 / 2}$ factor.

That the cavity state evolves when no photon is recorded by Bob might seem counterintuitive. Recall however that a null measurement provides information on the system and, hence, modifies its state. Recording no photon during $\tau$ is an indication that the number of photons is more likely to be small. The pseudo-Hamiltonian describes the reduction of the field energy associated to this information. We give below a more detailed insight into this evolution.

At the end of the time interval, the cavity is yet in a pure state, depending upon the measurement result recorded by Bob. A Monte Carlo trajectory is then defined by a series of random measurement results associated to successive time bins of duration $\tau$. At each step, the field state undergoes the action of either $\left[1-\kappa \tau a^{\dagger} a / 2\right]$ or $a$, depending upon the measurement result. It is computed by iterating the process, making a random decision according to the probability law (44) to determine whether $B$ is found in $\left|1^{(B)}\right\rangle$ or $\left|0^{(B)}\right\rangle$ at each stage. The state of $A$ at the beginning of the $(n+1)^{\text {th }}$ step is determined by the outcome of the $n^{\text {th }}$ measurement and $B$ is initialized to $\left|0^{(B)}\right\rangle$ at the beginning of each step.

The master equation result is recovered by assuming that Bob's measurements are left unread. The cavity mode ends up, at the end of the first time interval $\tau$, in the density operator averaged over the two possible outcomes:

$$
\rho(\tau)=p_{0}\left|\phi_{0}^{(A)}\right\rangle\left\langle\phi_{0}^{(A)}\left|+p_{1}\right| \phi_{1}^{(A)}\right\rangle\left\langle\phi_{1}^{(A)}\right| .
$$

Plugging in this equation the expressions (45) and (46) and keeping the first order terms in $\tau$, we get:

$$
\rho(\tau)-\left|\phi^{(A)}\right\rangle\left\langle\phi^{(A)}\left|=-\frac{\kappa \tau}{2}\left[a^{\dagger} a,\left|\phi^{(A)}\right\rangle\left\langle\phi^{(A)}\right|\right]_{+}+\kappa \tau a\right| \phi^{(A)}\right\rangle\left\langle\phi^{(A)}\right| a^{\dagger},
$$

where $[,]_{+}$is an anti-commutator. Identifying $\left(\rho(\tau)-\left|\phi^{(A)}\right\rangle\left\langle\phi^{(A)}\right|\right) / \tau$ with $d \rho / d t$, we recover the master equation (41).

The Monte-Carlo approach has many advantages. Assuming that Bob is observing the environment and communicating the results of his measurements to Alice means that she can, at all times, describe her system by a wavevector which evolves randomly, according to the unpredictable outcomes of the measurements. Calculating a set of Monte Carlo trajectories can be much more economical, for large photon numbers, or more generally for high-dimensionality systems, than solving the master equation.

Moreover, this approach is adapted to the description of a single realization of an experiment manipulating a unique quantum system. In many modern experiments, an information is continuously extracted from the system's environment and its wave function follows a random trajectory 
exhibiting explicit quantum jumps $[21,22,23]$. The statistics of these jumps is reproduced by a Monte Carlo simulation involving an environment with as many states as the number of possible exclusive measurements outcomes. In most cases, this number is small and a few quantum states of the environment detectors owned by Bob are enough to simulate the experimental results.

The Monte Carlo procedure is easily applied to the relaxation of a Fock state $|n\rangle$. Being an eigenstate of the pseudo-Hamiltonian, a Fock state does not evolve between quantum jumps. Each quantum jump is a transition between two adjacent Fock states. The cavity is always in a Fock state and the photon number undergoes a staircase evolution, with random jumps. The usual exponential decay is recovered by averaging many trajectories. We now turn to the interesting case of an initial coherent state. A Monte Carlo scenario for Schrödinger cat decoherence will be considered in the next Section.

\subsubsection{The coherent state paradox}

Let us describe the Monte Carlo trajectories starting from a coherent state $|\beta\rangle$. The probability for counting a photon in the first time interval is $p_{1}=\kappa \tau\left\langle\beta\left|a^{\dagger} a\right| \beta\right\rangle=\kappa \tau|\beta|^{2}=\kappa \tau \bar{n}$. If a photon is counted, the state is unchanged since $|\beta\rangle$ is an eigenstate of the jump operator $\sqrt{\kappa} a$. An evolution occurs if no photon is recorded. The imaginary frequency contribution to the pseudo Hamiltonian produces a decrease of the state amplitude:

$$
|\beta\rangle \rightarrow\left|\beta e^{-\kappa \tau / 2}\right\rangle
$$

This situation is paradoxical, since the field looses energy only if no photons are counted! How comes that the state does not change when one photon has been lost? We have the combination of two effects, which tend to change the photon number in opposite directions. One the one hand, the loss of a quantum reduces the average energy in the cavity. On the other hand, the photon click provides an information about the state in which the field was just before the photon was emitted, which tends to increase the photon number.

To understand this point, let us ask a simple question: knowing that it has emitted a photon at a given time, what is the probability $p_{c}(n)$ that the cavity contained $n$ photons just before the click? If the photon emission probability were independent on $n, p_{c}(n)$ would be equal to $p(n)=e^{-\bar{n}} \bar{n}^{n} / n$ !, the photon number distribution in the coherent state. In fact, the probability for losing a photon is proportional to $n$. It follows that $p_{c}(n)=k n p(n)$, where $k=1 / \bar{n}$ is determined by normalization. Thus:

$$
p_{c}(n)=\frac{n p(n)}{\bar{n}}=e^{-\bar{n}} \frac{\bar{n}^{n-1}}{(n-1) !}=p(n-1) .
$$

For a coherent state, $p_{c}(n)$ is equal to $p(n-1)$, the unconditional probability that the initial field contains $n-1$ photons. The maximum of $p(n-1)$, hence of $p_{c}(n)$, occurs for $n=\bar{n}+1$. The knowledge that a click has occurred biases the photon number distribution before this click towards larger $n$ values, with a photon number expectation exceeding the a priori average value by one unit. The loss of the photon signaled by the click brings this number back down to $\bar{n}$. The two effects exactly cancel and the state of the field has not changed!

This is a peculiar property of coherent states. For non-poissonian fields with larger fluctuations, the effect could be even more counter-intuitive and result in an overall increase of the photon expectation number, just after the loss of a photon! This situation requires fluctuations in the initial field energy. It does not occur for a Fock state, which looses its energy in an intuitive way.

Why, now, are coherent states loosing their energy when no photon clicks are registered? If no photon is detected, it is more likely that the photon distribution has less photons that was a priori assumed. To consider an extreme situation, a coherent field has a small probability, $e^{-\bar{n}}$, for containing no photon at all. This is the probability that the detector will never click, however long one waits. The longer the period without click, the more likely it becomes that the field is effectively in vacuum. Its wave function evolves continuously, without jump, under the effect of the non-unitary evolution and ends up in $|0\rangle$ ! 
The insensitivity of coherent states to jumps make their Monte Carlo trajectories certain. To determine the state at time $t$, we have concatenate evolutions during the successive intervals between jumps $t_{1}, t_{2}, \ldots t_{i}, \ldots t_{N}$ adding up to $t$. Whatever the distribution of these intervals, the final state, $\left|\beta e^{-\kappa\left(t_{1}+t_{2}+\ldots .+t_{N}\right) / 2}\right\rangle=\left|\beta e^{-\kappa t / 2}\right\rangle$ remains pure: coherent states are impervious to entanglement with the environment. They are the 'pointer states' of cavity decoherence.

\section{Cat state generation and decoherence studies}

In the last Section, we described Schrödinger cat states, quantum superpositions of coherent components with different classical phases. These states are ideal for decoherence studies, since they are pretty well isolated from their environment, being stored in a high- $Q$ cavity. Moreover, their 'size', measured by the distance between the two components, can be varied from the microscopic range (single photon fields) to the mesoscopic one. We describe in this Section how the interaction between a single Rydberg atom and a coherent state can be harnessed to produce and probe Schrödinger cat states. We first give a brief description of the atom/field interaction.

\subsection{The atom/cavity system}

We consider a two-level atom whose upper level $|e\rangle$ is connected to level $|g\rangle$ by a dipole electric transition at angular frequency $\omega_{e g}$. This system is equivalent to a spin- $1 / 2$ evolving in an abstract space, with a magnetic field, oriented along the 'vertical' $Z$ axis, accounting for the energy difference between $|e\rangle$ and $|g\rangle$. These states correspond to the eigenstates of the spin along $Z$, which we denote by $|+\rangle$ and $|-\rangle$.

Any spin or atomic operator can be expanded over the basis made up of the three Pauli matrices $\sigma_{X}, \sigma_{Y}$ and $\sigma_{Z}$ together with the unity $\mathbb{1}$. The atomic Hamiltonian is $H_{a}=\hbar \omega_{e g} \sigma_{Z} / 2$. The atomic raising and lowering operators $\sigma_{ \pm}$are $\sigma_{ \pm}=\left(\sigma_{X} \pm i \sigma_{Y}\right) / 2$, with $\sigma_{+}|-\rangle=|+\rangle \sigma_{-}|+\rangle=|-\rangle$ $\sigma_{+}|+\rangle=0 \sigma_{-}|-\rangle=0$. These atomic excitation creation/annihilation operators have a fermionic commutation $\left\{\sigma_{-}, \sigma_{+}\right\}=1$, where $\{$,$\} denotes an anti-commutator. There is a clear analogy$ between $\sigma_{ \pm}$and the photon creation and annihilation operators.

The atomic dipole operator is:

$$
\mathbf{D}=d\left(\boldsymbol{\epsilon}_{a} \sigma_{-}+\boldsymbol{\epsilon}_{a}^{*} \sigma_{+}\right)
$$

where $\boldsymbol{\epsilon}$ is a complex unit vector describing the transition polarization. For the circular states with principal quantum numbers 51 and 50, the transition is $\sigma$-polarized and $d=1776$ atomic units, a remarkably large value due to the huge size of the Rydberg states.

The complete atom-cavity Hamiltonian writes:

$$
H=H_{a}+H_{c}^{\prime}+H_{a c},
$$

where $H_{a}$ and $H_{c}^{\prime}$ are the atom and cavity Hamiltonians. The coupling term, $H_{a c}$, is $-\mathbf{D} \cdot \mathbf{E}_{c}$, where $\mathbf{E}_{c}$ the electric field operator at the atomic location. The Hamiltonian $H$ has been introduced by Jaynes and Cummings [24] as an approximation to matter-field coupling in free space. It is only in a cavity that it provides a precise description of the atomic dynamics, since the interaction with a single mode dominates the evolution. is then:

We assume first that the atom is sitting at cavity centre $[f(\mathbf{r})=1]$. The coupling Hamiltonian

$$
H_{a c}=-d\left[\boldsymbol{\epsilon}_{a} \sigma_{-}+\boldsymbol{\epsilon}_{a}^{*} \sigma_{+}\right] \cdot i \mathcal{E}_{0}\left[\boldsymbol{\epsilon}_{c} a-\boldsymbol{\epsilon}_{c}^{*} a^{\dagger}\right] .
$$

Its expansion involves four terms. Two are proportional to $\sigma_{-} a$ and $\sigma_{+} a^{\dagger}$. The first corresponds to a transition from $|e\rangle$ to $|g\rangle$, together with the annihilation of a photon. The second describes the emission of a photon by an atom in a transition from $|g\rangle$ to $|e\rangle$. When the cavity and atomic transition frequencies, $\omega_{c}$ and $\omega_{e g}$, are comparable, these terms correspond to non-resonant processes. They can be neglected (Rotating Wave Approximation). The two others, proportional to $\sigma_{+} a$ and $\sigma_{-} a^{\dagger}$, correspond to photon absorption or emission and the atom-cavity coupling reduces to:

$$
H_{a c}=-i \hbar \frac{\Omega_{0}}{2}\left[a \sigma_{+}-a^{\dagger} \sigma_{-}\right]
$$


where we introduce the 'single photon Rabi frequency', $\Omega_{0}$ :

$$
\Omega_{0}=2 \frac{d \mathcal{E}_{0} \epsilon_{a}^{*} \cdot \boldsymbol{\epsilon}_{c}}{\hbar} .
$$

We assumed that $\boldsymbol{\epsilon}_{a}^{*} \cdot \boldsymbol{\epsilon}_{c}$ is real and positive, hence $\Omega_{0}$. The frequency $\Omega_{0}$ measures the strength of the atom-field coupling. It is proportional to the interaction energy of the atomic dipole with a classical field corresponding to a single photon stored in $C$. In our experiments $\Omega_{0}=2 \pi \times 50$ $\mathrm{kHz}$, a rather large value. It is much larger than the cavity relaxation rate, achieving the 'strong coupling regime' of cavity QED.

The 'uncoupled states', eigenstates of $H_{a}+H_{c}^{\prime}$, are the tensor products $|e, n\rangle$ and $|g, n\rangle$ of atomic energy eigenstates and cavity Fock states, with the energies $\hbar\left(\omega_{e g} / 2+n \omega_{c}\right)$ and $\hbar\left(-\omega_{e g} / 2+\right.$ $\left.n \omega_{c}\right)$. The ground state of $H_{a}+H_{c}^{\prime}$ is the non-degenerate $|g, 0\rangle$ state. When the atom-cavity detuning, $\Delta_{c}=\omega_{e g}-\omega_{c}$, is much smaller than the atomic frequency, the uncoupled states $|e, n\rangle$ and $|g, n+1\rangle$ are degenerate $\left(\Delta_{c}=0\right)$ or nearly degenerate. They form a ladder of equally-spaced two-level manifolds, which are not coupled by $H_{a c}$. The diagonalization of the full Hamiltonian amounts to solving separate two-level problems.

\subsubsection{Eigenenergies and eigenvectors}

We consider here the restriction $H_{n}$ of the Jaynes and Cummings Hamiltonian to the manifold spanned by $|e, n\rangle$ and $|g, n+1\rangle$. Taking the energy reference at the mid-point between these levels, $H_{n}$ writes in matrix form:

$$
H_{n}=\frac{\hbar}{2}\left(\begin{array}{cc}
\Delta_{c} & -i \Omega_{n} \\
i \Omega_{n} & -\Delta_{c}
\end{array}\right),
$$

where

$$
\Omega_{n}=\Omega_{0} \sqrt{n+1} .
$$

The Rabi frequency $\Omega_{n}$ is proportional to $\sqrt{n+1}$, relative field amplitude in the $n$-photons Fock state.

The eigenvalues of $H_{n}$ are :

$$
E_{n}^{ \pm}= \pm \frac{\hbar}{2} \sqrt{\Delta_{c}^{2}+\Omega_{n}^{2}}
$$

and the eigenvectors, the 'dressed states':

$$
| \pm, n\rangle=\cos \theta_{n}^{ \pm}|e, n\rangle+i \sin \theta_{n}^{ \pm}|g, n+1\rangle,
$$

are generally entangled atom-cavity states. The mixing angles $\theta_{n}^{ \pm}$are given by

$$
\tan \theta_{n}^{ \pm}= \pm \frac{\sqrt{\Delta_{c}^{2}+\Omega_{n}^{2}} \mp \Delta_{c}}{\Omega_{n}} .
$$

The positions of the dressed energies are represented as a function of $\Delta_{c}$ on Figure 10. For large detunings, the dressed energies almost coincide with the uncoupled ones $\pm \hbar \Delta_{c} / 2$. At zero detuning, the uncoupled levels cross. The atom-cavity coupling transforms this crossing into an avoided crossing, the minimum distance between the dressed states being $\hbar \Omega_{n}$. We examine now two limiting cases: the resonant case $\left(\Delta_{c}=0\right)$ and the detuned case $\left(\Delta_{c} \gg \Omega\right)$.

\subsubsection{Resonant coupling}

In the resonant case, the mixing angles are $\theta_{n}^{ \pm}= \pm \pi / 4$ and:

$$
| \pm, n\rangle=\frac{1}{\sqrt{2}}[|e, n\rangle \pm i|g, n+1\rangle] \text {. }
$$

The separation of the dressed states at resonance, $\hbar \Omega_{n}$, corresponds to the frequency of the atomfield reversible energy exchange. We consider an atom in state $|e\rangle$ at time $t=0$ inside the cavity containing $n$ photons. The initial state, $\left|\Psi_{e}(0)\right\rangle=|e, n\rangle$, expands on the dressed states basis as:

$$
\left|\Psi_{e}(0)\right\rangle=\frac{1}{\sqrt{2}}[|+, n\rangle+|-, n\rangle]
$$




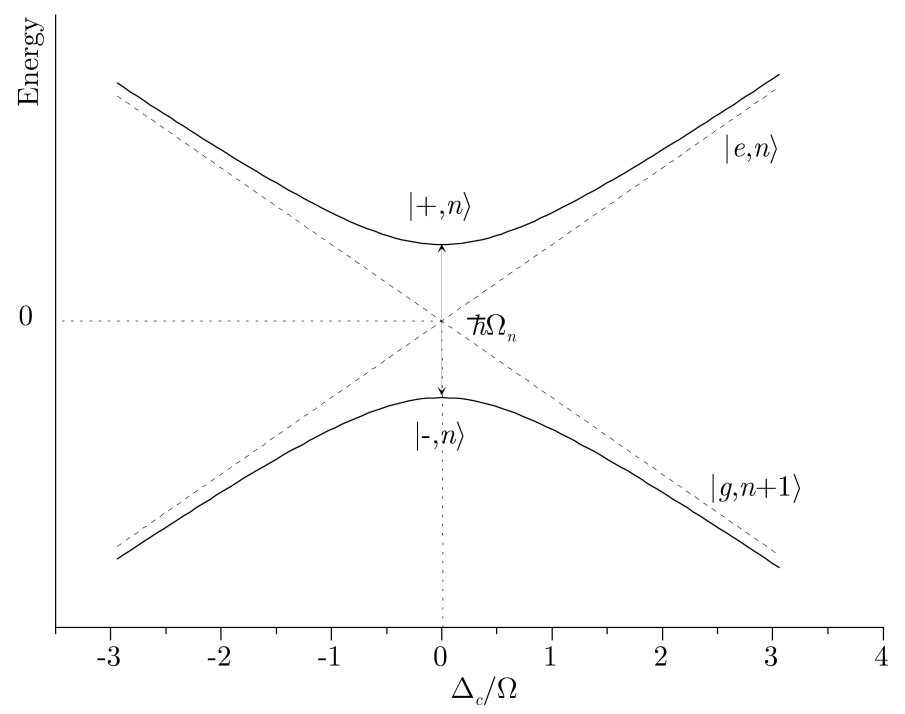

Figure 10: Dressed states energies as a function of the atom-cavity detuning $\Delta_{c}$. The uncoupled states energies are represented as dotted lines.

and becomes at time $t$ :

$$
\left|\Psi_{e}(t)\right\rangle=\frac{1}{\sqrt{2}}\left[|+, n\rangle e^{-i \Omega_{n} t / 2}+|-, n\rangle e^{i \Omega_{n} t / 2}\right] .
$$

The probabilities for finding the atom in $|e\rangle$ or $|g\rangle$ are obtained by returning to the uncoupled basis :

$$
\left|\Psi_{e}(t)\right\rangle=\cos \frac{\Omega_{n} t}{2}|e, n\rangle+\sin \frac{\Omega_{n} t}{2}|g, n+1\rangle .
$$

The coupling results in a reversible exchange between $|e, n\rangle$ and $|g, n+1\rangle$ at frequency $\Omega_{n}$. This is the Rabi oscillation phenomenon. It can be understood as a 'quantum beat' between the two dressed states [see Eq.(63)]. For the initial state $|e, 0\rangle$ (or $|g, 1\rangle$ ), this oscillation occurs at the frequency $\Omega_{0}$.

\subsubsection{Non-resonant atom/cavity coupling}

We give here a perturbative treatment valid for large detunings [25, 26]. We assume $\Delta_{c}=\omega_{e g}-\omega_{c}>$ 0 , with $\theta_{n} \ll 1$. We develop the eigenstates and eigenenergies in powers of $\Omega_{0} \sqrt{n+1} / \Delta_{c}$. To first order for states and to second order for energies:

$$
\begin{aligned}
& |+, n\rangle \approx|e, n\rangle+\frac{\Omega_{0} \sqrt{n+1}}{2 \Delta_{c}}|g, n+1\rangle \\
& |-, n\rangle \approx-|g, n+1\rangle+\frac{\Omega_{0} \sqrt{n+1}}{2 \Delta_{c}}|e, n\rangle \\
& \frac{1}{\hbar} E_{+, n}=n \omega_{c}+\frac{\omega_{e g}}{2}+\frac{\Omega_{0}^{2}(n+1)}{4 \Delta_{c}} \\
& \frac{1}{\hbar} E_{-, n}=(n+1) \omega_{c}-\frac{\omega_{e g}}{2}-\frac{\Omega_{0}^{2}(n+1)}{4 \Delta_{c}} .
\end{aligned}
$$

The dressed states $|+, n\rangle$ and $|-, n\rangle$ are very close to the uncoupled states, which are slightly 'contaminated' by the atom-field coupling. The energies of these levels are shifted to second order by an amount proportional to $n+1$, combination of a light shift, proportional to $n$, and of a Lamb shift effect. 
Assume now that we couple an atom in level $|e\rangle$ with a coherent field $|\alpha\rangle$ and let the two systems interact for a time $t$. Expanding the coherent state on a Fock state basis and taking into account that $|e, n\rangle$ is very close to the $|+, n\rangle$ dressed state, we get:

$$
\begin{aligned}
\left|\Psi_{e, \alpha}(0)\right\rangle & =|e\rangle|\alpha\rangle=\sum_{n} c_{n}|e, n\rangle \Rightarrow \\
\left|\Psi_{e, \alpha}(t)\right\rangle & \approx \sum_{n} c_{n} e^{-i n \omega_{c} t} e^{-i \omega_{e g} t / 2} e^{-i \Omega_{0}^{2}(n+1) t / 4 \Delta_{c}}|e, n\rangle,
\end{aligned}
$$

which, in interaction picture yields:

$$
\left|\widetilde{\Psi}_{e, \alpha}(t)\right\rangle \approx \sum_{n} c_{n} e^{-i \Omega_{0}^{2}(n+1) t / 4 \Delta_{c}}|e, n\rangle=e^{-i \Omega_{0}^{2} t / 4 \Delta_{c}}|e\rangle \otimes\left|\alpha e^{-i \Omega_{0}^{2} t / 4 \Delta_{c}}\right\rangle
$$

Similarly, for an atom initially in level $|g\rangle$ we obtain:

$$
\left|\widetilde{\Psi}_{g, \alpha}(t)\right\rangle \approx \sum_{n} e^{+i \Omega_{0}^{2} n t / 4 \Delta_{c}}|g, n\rangle=|g\rangle \otimes\left|\alpha e^{+i \Omega_{0}^{2} t / 4 \Delta_{c}}\right\rangle
$$

The cavity is in a coherent state, phase shifted by an angle $\pm \Phi_{0}= \pm \Omega_{0}^{2} t / 4 \Delta_{c}$ depending upon the atomic state. This effect is interpreted by attributing to the atom an index $N_{i}=1 \pm \Omega_{0}^{2} / 4 \Delta_{c} \omega_{c}$, with the + and - signs corresponding respectively to an atom in $|e\rangle$ or $|g\rangle$. With the parameters of our experiment we find, for $\Delta_{c}=3 \Omega,\left|N_{i} 1\right|=10^{-7}$, a huge value for a single atom effect. Note that this index is linear for extremely low fields only and saturates for average photon numbers of the order of $\left(\delta / \Omega_{0}\right)^{2}$, since the dispersive regime condition is no longer fulfilled. Note also the global quantum phase shift of the system's state when the atom is in $|e\rangle$. This is a cavity Lamb shift effect.

Up to now, we have considered the atom as motionless at cavity center. In the actual experiment, the atom flies across the cavity mode waist $w$. The atom-field coupling, proportional to the relative mode amplitude $f$, is thus a time-dependent quantity. In the dispersive regime, the previous results still hold, when the time $t$ is replaced by an effective interaction time $t_{i}^{d}$ taking into account the integrated atom-field coupling. For a full crossing of the mode:

$$
t_{i}^{d}=\sqrt{\frac{\pi}{2}} \frac{w}{v} .
$$

Note that the same approach can be used in the resonant case, with a different effective interaction time $t_{i}^{r}$ :

$$
t_{i}^{r}=\sqrt{\pi} \frac{w}{v}
$$

\subsection{Dispersive cat generation}

The phase shift produced by a single atom on a coherent state takes opposite values for levels $|e\rangle$ and $|g\rangle$. Phase shift values as large as a few radians can be reached. The ability of a single atom to shift by a macroscopic angle the phase of a field containing many quanta provides an ideal tool for experiments with cat states. When an atom is sent across the cavity in a superposition of $|e\rangle$ and $|g\rangle$, a mesoscopic cat is produced in the cavity.

\subsubsection{Reading the phase imprint of a single atom}

We first measure these phase shifts. The usual method to measure the phase of a coherent 'signal' in quantum optics is homodyning: the signal is mixed with a reference of same frequency and variable phase $\varphi$. The phase distribution of the signal is obtained by measuring the intensity of the mixed signal + reference as a function of $\varphi$.

We have adapted this general method to our set-up and measured by homodyning the phase shift induced by a single atom on a coherent field [27]. The experimental sequence involves two 
successive field injections in the cavity, and two atoms. First, a signal field with amplitude $\beta$ is injected by connecting the cavity to the source $S$ for $2 \mu \mathrm{s}$. The modulus of $\beta$ is calibrated in an independent experiment, using the light shift on a non-resonant atom [28]. An off-resonant index atom $A_{i}$ (atom-cavity detuning $\Delta_{c}$ ), prepared with velocity $v$ in level $|e\rangle$ or $|g\rangle$, is then sent across the cavity and imprints its phase shift $\pm \Phi_{0}$ on the field whose amplitude becomes $\beta \exp \left( \pm i \Phi_{0}\right)$.

The $\Phi_{0}$ value is adjusted by an independent control of the $\Delta_{c}$ and $v$ parameters. The detuning $\Delta_{c}$ is tunedfrom a few tens to a few hundred $\mathrm{kHz}$ by Stark-shifting the atomic transition frequency. A second reference field injection follows before the cavity field has had time to appreciably decay. This reference has a complex amplitude $-\alpha=-\beta \exp (i \varphi)$. Its modulus is the same as the one of the signal field, but its phase $\varphi$ can be continuously adjusted.

To probe the final cavity field, we use a second atom, $A_{p}$. This atom is sent in $|g\rangle$. It is tuned at resonance with the mode and absorbs the homodyned field. The information provided by the probe atom absorption is used, in a repetitive experiment, to reconstruct the $Q$ function of the phase-shifted field [16]. Let us recall that $Q(\alpha)$ is $1 / \pi$ times the probability $p(0)$ for finding the cavity in the vacuum, after the field has been translated by the amplitude $-\alpha$ [see Eq.(34) in Section 2.4]. This translation is realized by reference field injection.

Ideally, we want a dichotomic signal indicating whether the final number of photons is zero or not. Let us call $P_{e}(n)$ the probability for finding $A_{p}$ in $|e\rangle$ when there are $n$ photons in $C$. Obviously $P_{e}(0)=0$. A dichotomic signal would be obtained if $P_{e}(n)$ were different from 0 and independent of $n$ for all $n \geq 1$. This is not the case for an arbitrary atom-field effective interaction time $t_{i}^{r}$, since the atom undergoes a Rabi oscillation whose period is $n$-dependent.

It is nevertheless possible to select an optimal effective interaction time $t_{i}^{r}=t_{\text {opt }}=5 \pi / 2 \Omega_{0}$, for which $P_{e}(1), P_{e}(2)$ and $P_{e}(3)$ are approximately equal to $1 / 2$. For larger $n$ 's, this condition is not fulfilled for an ideal quantum Rabi oscillation. However, experimental imperfections wash out the oscillations for large photon numbers. Practically, we also get, to a good approximation, $P_{e}(n)=1 / 2$ for $n>3$. Hence, the probability $P_{e}$ for detecting $A_{p}$ in $|e\rangle$ is $P_{e}=[1-p(0)] / 2$ and $Q(\alpha)=\left(1-2 P_{e}\right) / \pi$. The variation of $P_{e}$ when $\varphi$ is swept determines directly $Q$ along a circle of radius $|\beta|$. The experiment is performed with an index atom $A_{i}$ either in $|e\rangle$ or in $|g\rangle$ or, for reference, without sending $A_{i}$. The experimental signals are shown in Figure $11\left(\Delta_{c} / 2 \pi=50 \mathrm{kHz}\right.$, $v=200 \mathrm{~m} / \mathrm{s}$ ). The coherent field contains 29 photons in average. The open circles represent $Q$ when $A_{i}$ is not sent. This peak serves as a reference for the signals shown as black circles and black squares, which correspond respectively to $A_{i}$ in level $|e\rangle$ or $|g\rangle$. The observed phase shift, $\pm \Phi_{0}= \pm 39^{\circ}$ is in agreement with the theoretical expectations.

\subsubsection{Trapping a cat in an atomic interferometer}

This single-atom index effect can be exploited to generate cat states of the field. We merely have to cast $A_{i}$, prepared initially in $|e\rangle$, in a superposition of states $|e\rangle$ and $|g\rangle$ by a classical microwave pulse $R_{1}$ before $A_{i}$ enters the mode. The field then acquires two distinct classical phases at once and gets entangled with the atom. This entanglement can be analyzed by finally detecting the atomic state after the atom exits $C$. This analysis is carried out by combining a second microwave pulse $R_{2}$ with a state selective detector $D$.

Let us follow the successive stages of this experiment. The atom is prepared by $R_{1}$ in the superposition $(|e\rangle+|g\rangle) / \sqrt{2}$. After it has crossed $C$, the atom-field system evolves into the entangled state:

$$
\left|\Psi_{1}\right\rangle=\frac{e^{-i \Phi_{0}}}{\sqrt{2}}|e\rangle \otimes\left|\beta e^{-i \Phi_{0}}\right\rangle+\frac{1}{\sqrt{2}}|g\rangle \otimes\left|\beta e^{i \Phi_{0}}\right\rangle .
$$

This is a typical quantum measurement situation, in which a large 'meter' (the field) points simultaneously towards different directions corresponding to two orthogonal states of a microscopic system $\left(A_{i}\right)$. If $A_{i}$ were directly detected at the cavity exit in $|e\rangle$ or $|g\rangle$, the field would be projected in the corresponding coherent state and the quantum ambiguity lost. The atom's measurement would tell us whether the dispersive index had taken one value or the other.

The second microwave pulse $R_{2}$ can be used to preserve the blurred state of the meter by 


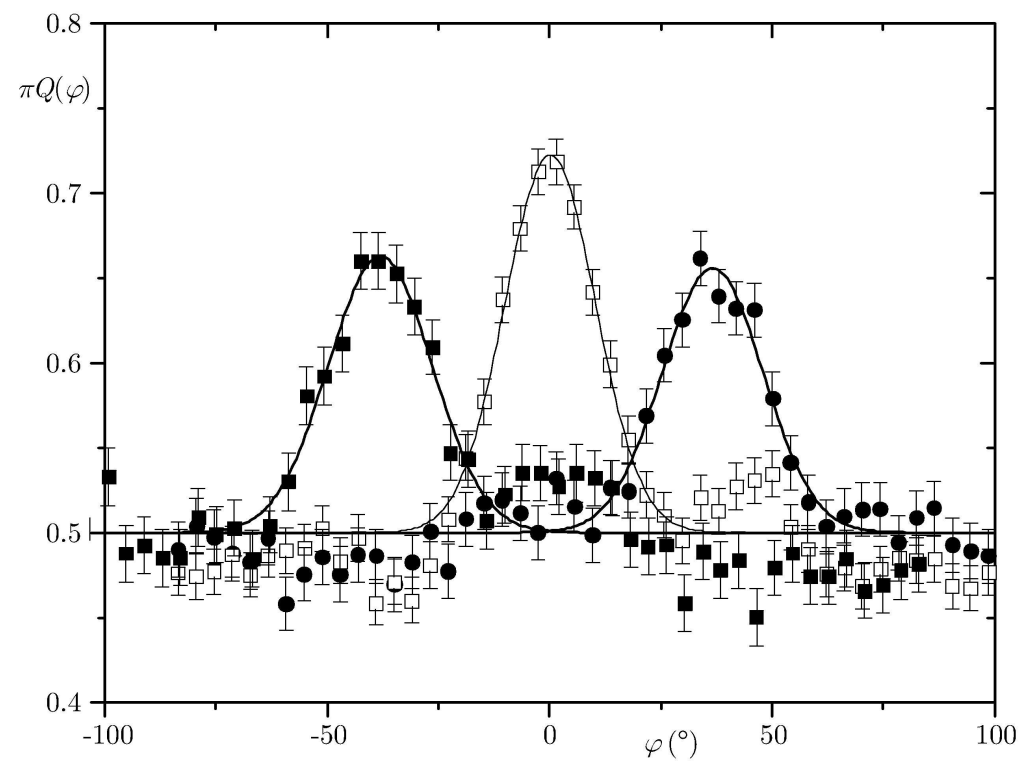

Figure 11: Phase-shift of a coherent field. Field phase distribution $\pi Q(\varphi)$ as a function of the displacement phase $\varphi$ in degrees. Open squares: reference 29 photons coherent field. Solid circles (squares): phase distribution after interaction with an atom in $|e\rangle(|g\rangle)$. The error bars depict statistical fluctuations. The solid lines are Gaussian fits.

erasing the information about the atomic index. Let us apply in $R_{2}$ the transformations:

$$
|e\rangle \rightarrow \frac{1}{\sqrt{2}}\left(|e\rangle+e^{i \chi}|g\rangle\right) \quad ; \quad|g\rangle \rightarrow \frac{1}{\sqrt{2}}\left(|g\rangle-e^{-i \chi}|e\rangle\right)
$$

where $\chi$ can be adjusted freely by setting the relative phase of the $R_{1}$ and $R_{2}$ pulses. Immediately after $R_{2}$, the combined atom-field state is:

$$
\begin{aligned}
\left|\Psi_{2}\right\rangle=\frac{1}{2}|e\rangle \otimes\left[e^{-i \Phi_{0}}\left|\beta e^{-i \Phi_{0}}\right\rangle-e^{-i \chi}\left|\beta e^{i \Phi_{0}}\right\rangle\right]+ \\
+\frac{1}{2}|g\rangle \otimes\left[e^{i\left(\chi-\Phi_{0}\right)}\left|\beta e^{-i \Phi_{0}}\right\rangle+\left|\beta e^{i \Phi_{0}}\right\rangle\right] .
\end{aligned}
$$

The two states $|e\rangle$ and $|g\rangle$ are now correlated to two cat states, mutually orthogonal when $\Phi_{0}>$ $1 / \sqrt{\bar{n}}$. The phase $\chi$ of the second Ramsey zone can be adjusted to simplify a little bit the expressions of the cat states. Setting $\chi=\Phi_{0}$, we get:

$$
\left|\Psi_{2}\right\rangle=\frac{e^{-i \Phi_{0}}}{2}|e\rangle \otimes\left[\left|\beta e^{-i \Phi_{0}}\right\rangle-\left|\beta e^{i \Phi_{0}}\right\rangle\right]+\frac{1}{2}|g\rangle \otimes\left[\left|\beta e^{-i \Phi_{0}}\right\rangle+\left|\beta e^{i \Phi_{0}}\right\rangle\right] .
$$

This equation shows that the final detection of the atom projects with equal probabilities the field into one of the two cat states $\left[\left|\beta e^{-i \Phi_{0}}\right\rangle \pm\left|\beta e^{i \Phi_{0}}\right\rangle\right] / \sqrt{2}$. When $\Phi_{0}=\pi / 2$, these states become the even and odd $\pi$-phase cat states [Eq.(24) and (29)]:

$$
\left|\Psi_{2}\right\rangle=-\frac{i}{2}|e\rangle \otimes[|\gamma\rangle-|-\gamma\rangle]+\frac{1}{2}|g\rangle \otimes[|\gamma\rangle+|-\gamma\rangle]
$$

where $\gamma=-i \beta$. It is impossible to predict the field state eventually obtained, which is revealed only by the outcome of the atomic measurement.

We shall describe later how this cat can be probed by a 'quantum mouse'. Before that, the simple detection of the index atom $A_{i}$ provides some information about the cat generation and 
sheds an intersting light onto this experiment. What are then the probabilities for detecting $A_{i}$ in either levels?

Let us suppose first that we apply onto $A_{i}$ only the $R_{1}$ and $R_{2}$ pulses. We recognize a Ramsey atomic interferometer [29], widely used in high-precision atomic clocks. The probability, $P_{g}$, for observing finally the atom in level $|g\rangle$ exhibits modulations (Ramsey fringes) as a function of the relative pulse phase $\chi: P_{g}=(1+\cos \chi) / 2$. This modulation results from a quantum interference effect. When the atom is finally in $|g\rangle$, the transition may occur either during the first pulse, $R_{1}$, or during the second, $R_{2}$. These two quantum paths are indistinguishable and the corresponding probability amplitudes interfere.

When the cavity is inserted between $R_{1}$ and $R_{2}$, the interferometric signal provides an information about the atom-cavity interaction. We shall, first, consider the case of an empty cavity. By setting $\beta=0$ in Eq.(73), we obtain for the final atom-field system:

$$
\left|\Psi_{2}^{(\beta=0)}\right\rangle=\frac{e^{-i \Phi_{0}}}{2}\left[1-e^{-i\left(\chi-\Phi_{0}\right)}\right]|e, 0\rangle+\frac{1}{2}\left[1+e^{i\left(\chi-\Phi_{0}\right)}\right]|g, 0\rangle
$$

which yields the probabilities $P_{e}^{0}$ and $P_{g}^{0}=1-P_{e}^{0}$ for finding the atom in $|e\rangle$ or $|g\rangle$ :

$$
P_{e}^{0}=1-P_{g}^{0}=\frac{1-\cos \left(\chi-\Phi_{0}\right)}{2} .
$$

The fringes are phase shifted by an angle $\Phi_{0}$ with respect to their position if there were no cavity between $R_{1}$ and $R_{2}$. The phase shift of the atomic superposition between the two pulses reflects the transient Lamb shift of level $|e\rangle$ in the empty cavity mode (Section 3.1).

When the cavity contains a coherent field with $\bar{n}$ photons on average, this field undergoes different phase shifts, depending on the state of the atom between $R_{1}$ and $R_{2}$. The final cavity field amplitude measures the atomic state between the two pulses. We recognize here the ingredients of a 'which-path' experiment.

Such gedanken experiments have been discussed in the early days of quantum theory. A Young interferometer, for instance, produces fringes due to the interference between two quantum paths corresponding to its two slits. Bohr's complementarity states that these fringes are incompatible with any unambiguous information about the path actually followed by the particle in the interferometer.

We are thus discussing here a complementarity situation. If the two final cavity states are orthogonal, which occurs for $\Phi_{0}>1 / \sqrt{\bar{n}}$, they could, by the detection of their phase, tell away the path of the atom. This is enough to destroy the fringes. In more quantitative terms, the contrast of the fringes reveals the amount of entanglement between the atom and the field. When this entanglement is maximum, the fringes are fully suppressed. They have, on the other hand, a maximum contrast when the two systems are separable.

More precisely, the probabilities for finding the atom in $|e\rangle$ or $|g\rangle$ when the cavity contains a coherent field with $\bar{n}$ photons on average are obtained from Eq.(73):

$$
\begin{aligned}
P_{e}^{\bar{n}} & =1-P_{g}^{\bar{n}}=\frac{1}{2}\left\{1-\operatorname{Re}\left[e^{i\left(\Phi_{0}-\chi\right)}\left\langle\beta e^{-i \Phi_{0}} \mid \beta e^{i \Phi_{0}}\right\rangle\right]\right\} \\
& =\frac{1}{2}\left\{1-\cos \left[\chi-\Phi_{0}-\bar{n} \sin \left(2 \Phi_{0}\right)\right] e^{-\bar{n}\left[1-\cos \left(2 \Phi_{0}\right)\right]}\right\} .
\end{aligned}
$$

We recognize in the first line the scalar product of the two final field components, whose analytical expression is given in the last line. The argument $\bar{n}\left[1-\cos \left(2 \Phi_{0}\right)\right]$ of the last exponential in Eq.(78) represents the square of the distance in phase space of the two field components. The Ramsey fringes appear to be phase-shifted by an angle proportional to $\bar{n}$ and their amplitude is suppressed by a factor decreasing exponentially with the separation of the field components.

We have observed these Ramsey fringes for $\bar{n}=9.5$ and $t_{i}^{d}=19 \mu \mathrm{s}$ [7] for different phase splittings $\Phi_{0}$ obtained by varying $\Delta_{c}$. The signals for three different values of $\Phi_{0}$ are shown in Figure 12(a) with, in the insets, the final field states phase space representations. The collapse of the fringe amplitude when the field components separate is conspicuous. The fringe contrast 

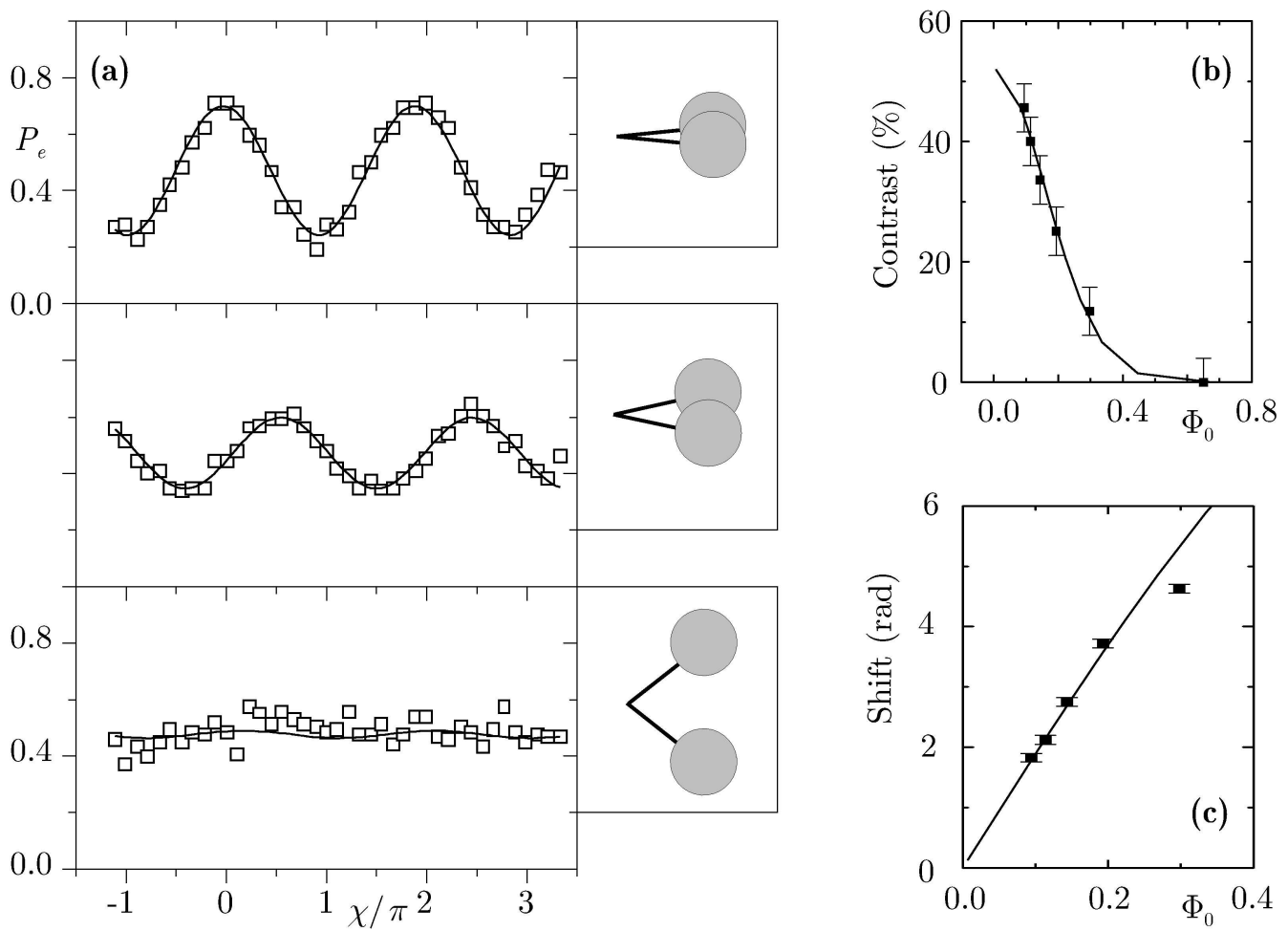

Figure 12: Schrödinger cat and complementarity. (a) Ramsey fringes for $\beta=3.1$ and three different $\Phi_{0}$ values $\left(0.1,0.2\right.$ and 0.69 radians, corresponding to $\Delta_{c} / 2 \pi=712,347$ and $104 \mathrm{kHz}$ respectively from top to bottom). The insets give a pictorial representation of the two field phase components. (b) Ramsey fringes contrast versus $\Phi_{0}$. The solid line corresponds to the theoretical predictions, scaled by the finite Ramsey interferometer contrast. (c) Fringes shift (in radians) versus $\Phi_{0}$. The slope of the fitted line provides a calibration of the photon number.

is shown versus $\Phi_{0}$ in Figure 12(b) and the fringe phase shift in Figure 12(c). In these plots, the points are experimental and the curves given by theory, with an overall contrast adjustment taking into account the imperfections of the Ramsey interferometer. Note that the theoretical formula (78), valid in the dispersive limit, does not apply for the smallest detuning $\Delta_{c}=104 \mathrm{kHz}$. An exact expression of the phase shifts based on the exact dressed states is used for the largest values of $\Phi_{0}$.

The experiment is found in excellent agreement with the theory. This is a clear indication that the field components are separated in phase space. The variation of the fringe phase with $\Phi_{0}$ reveals the light shift effect experienced by the atom. This variation [Figure 12(c)] yields the calibration of the photon number. Moreover, this experiment presents a direct illustration of the complementarity concept in a simple interferometer arrangement. Note that other complementarity tests have been performed with the same set-up [30].

\subsection{Decoherence of cavity cats}

Before coming to the detection of the cat coherence, we briefly describe its relaxation. We present three different approaches, which shed complementary lights. We give, first, the formal solution of the master equation. We present then an enlightening interpretation of its results, using again the complementarity concept. Finally, we discuss Monte Carlo trajectories for the field parity. For the sake of simplicity, we consider only the zero temperature case. 
(a)

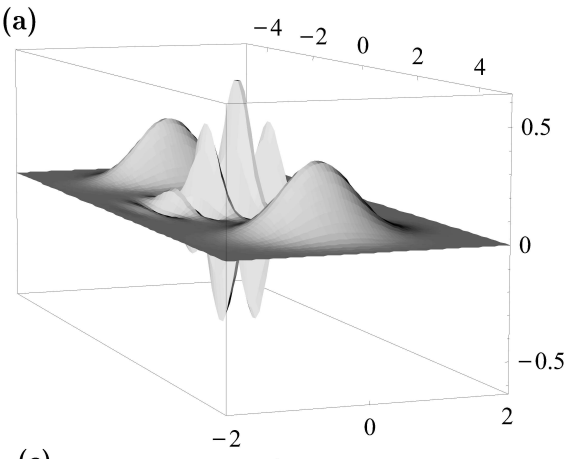

(c)

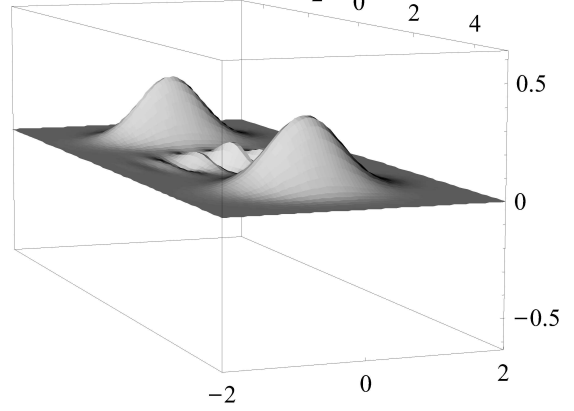

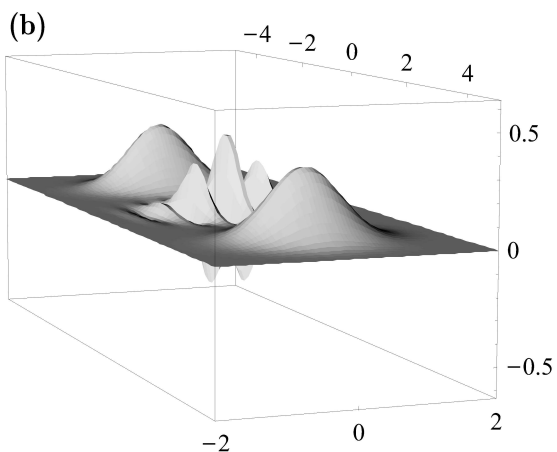

(d)

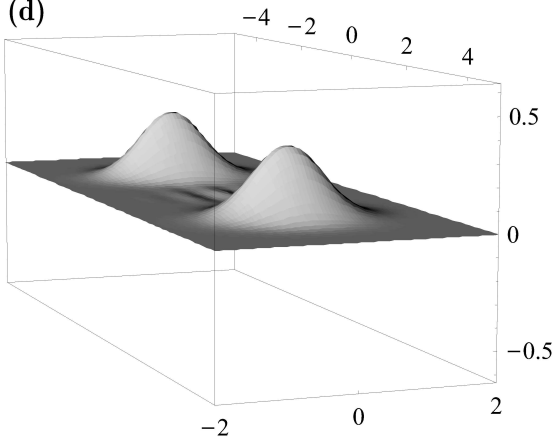

Figure 13: Evolution of the Wigner function of a cat state (10 photons on the average). (a) to (d): $t=0, t=T_{c} / 20, t=T_{c} / 5$ and $t=T_{c} / 2$ respectively.

\subsubsection{Solution of the master equation}

Under relaxation, a coherent state remains coherent, with an amplitude $\beta(t)=\beta e^{-\kappa t / 2}$ decaying exponentially with time. Its density operator reduces to a projector $\rho(t)=|\beta(t)\rangle\langle\beta(t)|$. Coherent states are 'pointer states' of the field, impervious to entanglement with the environment. It is convenient to study the evolution of an arbitrary state by expanding it on a coherent state basis. We do not recall here the derivation of the cat density operator versus time [31], leading to:

$$
\begin{aligned}
& \rho^{ \pm}(t)=\frac{1}{2}[|\beta(t)\rangle\langle\beta(t)|+|-\beta(t)\rangle\langle-\beta(t)| \pm \\
&\left. \pm e^{-2 \bar{n}\left(1-e^{-\kappa t}\right)}(|\beta(t)\rangle\langle-\beta(t)|+|-\beta(t)\rangle\langle\beta(t)|)\right] .
\end{aligned}
$$

The fast decay of the coherence terms is described by the exponential coefficient of the off-diagonal terms in Eq.(79). It decays at short times as $\exp (-2 \bar{n} \kappa t)$ and the coherent cat turns, for $t>1 / \bar{n} \kappa$, into a mixture of states. This relaxation process exhibits the essential features of the decoherence of a mesoscopic state superposition. It is much faster than the cavity energy relaxation and gets faster and faster when the 'size' of the cat, measured by the average photon number in the coherent components, increases.

This fast decoherence is pictorially exhibited by the Wigner functions, represented at four different times in Figure 13 for an even cat state containing $\bar{n}=10$ photons on average. The decoherence process appears clearly on these plots, which exhibit two very different time constants. After a very short time, the interference pattern near the origin, the 'cat whiskers', which are a signature of its coherence, have been washed out, leaving only the two Gaussian peaks, which slowly relax down to vacuum.

\subsubsection{Decoherence and complementarity}

The master equation approach does not provide a deep insight into decoherence mechanisms. We give now a heuristic derivation of Eq.(79), directly describing the environment evolution. This 
intuitive approach emphasizes the deep link between decoherence and complementarity [32].

A standard model for the environment $E$ is a bath of harmonic oscillators labeled by the index $i$, linearly coupled to $C$. Their frequencies span a wide range around $\omega_{c}$. Starting from a coherent state $|\beta\rangle$ at time $t=0$ and an empty environment, the global cavity-environment system evolves at time $t$ into a non-entangled state:

$$
\left|\Psi^{C E}(t)\right\rangle=|\beta(t)\rangle \otimes \prod_{i}\left|\epsilon_{i}(t)\right\rangle,
$$

where $\beta(t)=\beta e^{-\kappa t / 2}$ and $\epsilon_{i}(t)$ is the very small complex amplitude at time $t$ of the $i^{\text {th }}$ oscillator in $E$. Due to the linear coupling between $C$ and the environment oscillators, the phase of $\epsilon_{i}(t)$ is linearly related to the phase of $\beta$. Energy conservation requires moreover that the total number of quanta in $E$ equals the number of photons lost by $C$ :

$$
\sum_{i}\left|\epsilon_{i}(t)\right|^{2}=\bar{n}\left(1-e^{-\kappa t}\right) .
$$

Suppose now that we prepare at $t=0$ the field in a cat state:

$$
\left|\Psi_{\text {cat }}(0)\right\rangle=\frac{e^{i \psi_{1}}}{\sqrt{2}}\left|\beta e^{i \Phi}\right\rangle+\frac{e^{i \psi_{2}}}{\sqrt{2}}\left|\beta e^{-i \Phi}\right\rangle .
$$

The state of the $C+E$ system at time $t$ is obtained by superposing the contributions of the two parts of the cat, each being given by an expression of the form (80). Noting that, when $\beta$ is phase shifted by $\pm \Phi$, the same shift is experienced by the $\epsilon_{i}(t)$ amplitudes, we have:

$$
\begin{aligned}
\left|\Psi_{\text {cat }}^{C E}(t)\right\rangle= & \frac{e^{i \psi_{1}}}{\sqrt{2}}\left|\beta(t) e^{i \Phi}\right\rangle \otimes \prod_{i}\left|\epsilon_{i}(t) e^{i \Phi}\right\rangle+ \\
& +\frac{e^{i \psi_{2}}}{\sqrt{2}}\left|\beta(t) e^{-i \Phi}\right\rangle \otimes \prod_{i}\left|\epsilon_{i}(t) e^{-i \Phi}\right\rangle .
\end{aligned}
$$

The two parts of the cat are correlated to two states of $E$ :

$$
\left|E^{+}(t)\right\rangle=\prod_{i}\left|\epsilon_{i}(t) e^{i \Phi}\right\rangle,
$$

and

$$
\left|E^{-}(t)\right\rangle=\prod_{i}\left|\epsilon_{i}(t) e^{-i \Phi}\right\rangle,
$$

each of which is the product of minute copies of the cavity cat components disseminated in the environment. Each oscillator in $E$ is involved in a superposition of two states with a very small amplitude $\epsilon_{i}$ and a large phase splitting $2 \Phi$, a 'Schrödinger kitten' so to speak. The states $\left|E^{+}(t)\right\rangle$ and $\left|E^{-}(t)\right\rangle$ get quickly mutually orthogonal. Using Eqs.(22) and (81), we find:

$$
\begin{aligned}
\left\langle E^{-}(t) \mid E^{+}(t)\right\rangle & =\exp \left[-\sum_{i}\left|\epsilon_{i}(t)\right|^{2}\left(1-e^{2 i \Phi}\right)\right] \\
& =\exp \left[-\bar{n}\left(1-e^{-\kappa t}\right)\left(1-e^{2 i \Phi}\right)\right],
\end{aligned}
$$

an expression which goes very rapidly to zero as $t$ increases. The fast loss of coherence is a complementarity effect. It is a matter of information about the cat state leaking into $E$. The final states of each oscillator in $E$ have a near unity overlap (since $\left|\epsilon_{i}\right|^{2} \ll 1$ ), but there are very many of them and the product of their scalar products quickly vanishes.

In other words, an information about the phase $\pm \Phi$ of the cat state components could not be obtained from a single environment oscillator whose amplitude is very small and hence phase fluctuations very large. It could however be recovered, at least in principle, from a measurement 
of the environment as a whole, combining small amounts of information disseminated among the elementary oscillators. This is enough to kill the cat coherence and the interference effects associated to it. When tracing over $E$, the cat coherence is multiplied by $\left\langle E^{-}(t) \mid E^{+}(t)\right\rangle$ and $\rho(t)$ finally writes:

$$
\begin{aligned}
& \rho(t)=\frac{1}{2}\left\{\left|\beta(t) e^{i \Phi}\right\rangle\left\langle\beta(t) e^{i \Phi}|+| \beta(t) e^{-i \Phi}\right\rangle\left\langle\beta(t) e^{-i \Phi}\right|+\right. \\
& \left.+\left\langle E^{-}(t) \mid E^{+}(t)\right\rangle e^{i\left(\psi_{1}-\psi_{2}\right)}\left|\beta(t) e^{i \Phi}\right\rangle\left\langle\beta(t) e^{-i \Phi}\right|+\text { h.c. }\right\} .
\end{aligned}
$$

Setting $\Phi=\pi / 2$ and $\psi_{1}-\psi_{2}=0$ or $\pi$, we recover, as expected, Eq.(79) giving the evolution of an even or odd $\pi$-phase cat.

\subsubsection{Parity jumps of a $\pi$-phase cat}

We now turn to the Monte Carlo picture. Let us imagine that we use the set-up of Figure 9 to follow the evolution of a field initially prepared in the even cat state $\left|\Psi_{\text {cat }}^{+}(0)\right\rangle=[|\beta\rangle+|-\beta\rangle] / \sqrt{2}$. The photon clicks are counted in successive time bins of duration $\tau$. Until the first click at time $t_{1}$, the non-unitary evolution simply shrinks the amplitude of the cat components, turning $\beta$ into $\beta\left(t_{1}\right)=\beta e^{-\kappa t_{1} / 2}$. The cat state is continuously changed into $\left|\Psi_{\text {cat }}^{+}\left(t_{1}\right)\right\rangle=\left[\left|\beta\left(t_{1}\right)\right\rangle+\left|-\beta\left(t_{1}\right)\right\rangle\right] / \sqrt{2}$.

The first click corresponds to a cavity state quantum jump, described by the annihilation operator $a$. According to Eq.(31), the cat parity suddenly switches, the field state jumping from $\left|\Psi_{\text {cat }}^{+}\left(t_{1}\right)\right\rangle$ to $\left|\Psi_{\text {cat }}^{-}\left(t_{1}\right)\right\rangle=\left[\left|\beta\left(t_{1}\right)\right\rangle-\left|-\beta\left(t_{1}\right)\right\rangle\right] / \sqrt{2}$. The shrinking of the cat components then resumes until a second jump restores the initial parity and so on. As long as the field energy has not appreciably decayed, the probability of occurrence of a click in a time bin is $p_{1}=\kappa \bar{n} \tau$ and the average duration between clicks is $\tau_{D}=1 /(\kappa \bar{n})$. On each Monte Carlo trajectory, a fast random parity jumping is combined with a slow deterministic shrinking of the amplitude. The characteristic rates of these evolutions are in the ratio $\bar{n}$.

The information provided by a click has an immediate effect on a field in a superposition of two coherent states, even if each of them, prepared separately in $C$, would remain unchanged. This is a genuine quantum effect. The information provided by the click changes the relative quantum phase between the two parts of the field wave-function and, hence, its parity.

Assuming that the photon counter is perfect, the parity of the number of clicks recorded until time $t$ would tell us without ambiguity whether the field is in the state $\left|\Psi_{\text {cat }}^{+}(t)\right\rangle$ or $\left|\Psi_{\text {cat }}^{-}(t)\right\rangle$. We could thus follow a Monte Carlo trajectory in which the field would be a perfect, albeit random cat state. Observing the environment makes it thus possible, at least in principle, to keep the cat coherence alive for a time of the order of $T_{c}=1 / \kappa$. This continuous observation does not give us the power, though, to choose at a given time the parity of the cat. We can merely record it.

Monitoring the environment is of course but a gedanken experiment. It is more realistic, if extremely difficult, to keep the cat's coherence by monitoring the cavity itself. As we shall see in the next Section, the cavity photon number parity can be measured non-destructively [33]. A stream of probe atoms could then record in real time the decaying cat's parity jumps.

In this ideal situation, the action of these jumps may be, to some extent, canceled. Whenever the cat parity changes, an atom is sent into the cavity, interacts resonantly with it and emits a photon in the mode, restoring the parity. Numerical simulations show that a cat state's coherence could be preserved, by this quantum feedback procedure, over times of the order of $T_{c}[34,35]$

As soon as we give up to monitor parity jumps, the field is described by a density operator $\rho$, obtained by averaging Monte Carlo trajectories. After a time of the order of $\tau_{D}=1 / \bar{n} \kappa$ there are statistically as many trajectories with even or odd click numbers. The density operator is then the sum with equal weights of projectors on even and odd cats:

$$
\rho_{A}\left(t>\tau_{D}\right) \sim \frac{1}{2}\left|\Psi_{\text {cat }}^{+}(t)\right\rangle\left\langle\Psi_{\text {cat }}^{+}(t)\left|+\frac{1}{2}\right| \Psi_{\text {cat }}^{-}(t)\right\rangle\left\langle\Psi_{\text {cat }}^{-}(t)\right|
$$

It can be equivalently expressed as an incoherent sum with equal weights of projectors on the $|\beta(t)\rangle$ and $|-\beta(t)\rangle$ states. We thus retrieve the result predicted by an exact solution of the master equation. 


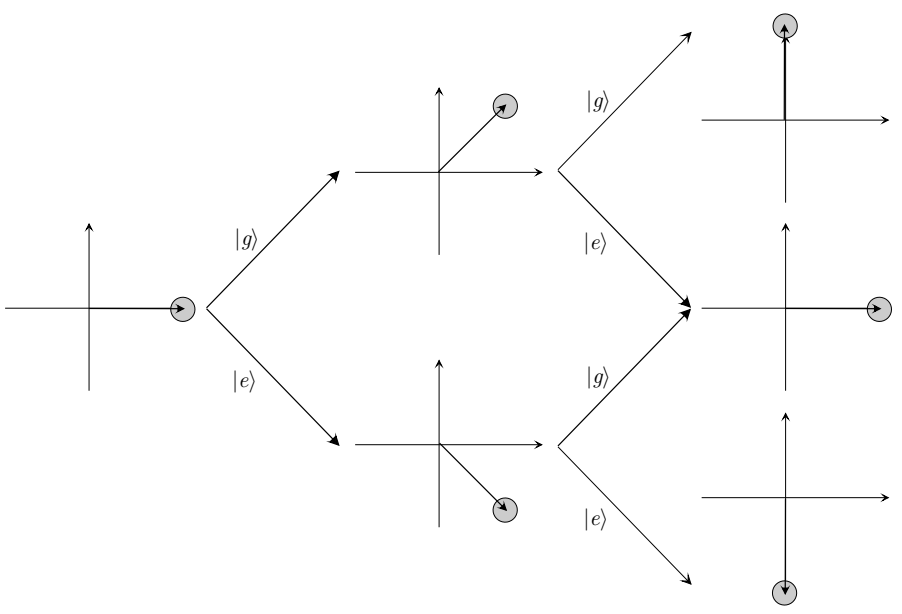

Figure 14: The interfering paths in the two atom experiment.

\subsection{Probing the cat with a quantum mouse}

The detection of the index atom $A_{i}$ leaves in $C$ a Schrödinger cat. How can we probe its fast relaxation towards a statistical mixture of coherent components? We must rely on an atomic signal to get an indirect information about the field. After some free evolution time, we probe $C$ with a second atom, $A_{p}$. Elaborating on Schrödinger's metaphor, $A_{p}$ is a 'quantum mouse' sent in $C$ to probe the coherence of the relaxing cat state left by $A_{i}$.

Let us first discuss this experiment in simple qualitative terms. Atom $A_{i}$, interacting with the initial coherent state $|\beta\rangle$, leaves in $C$ a quantum superposition of $\left|\beta \exp i \Phi_{0}\right\rangle$ and $\left|\beta \exp -i \Phi_{0}\right\rangle$. The mouse $A_{p}$ undergoes the same transformations as $A_{i}$ in $R_{1}$ and $R_{2}$ and produces the same quantum superposition of phase shifts on the cavity field. Each of the phase components left by $A_{i}$ is thus again split in two parts dephased by $\pm \Phi_{0}$. This process is pictorially represented in Figure 14 .

The cavity finally contains a three-components cat. The phases $\pm 2 \Phi_{0}$ correspond to the situations in which $A_{i}$ and $A_{p}$ crossed $C$ in the same state $[(|e\rangle,|e\rangle)$ or $(|g\rangle,|g\rangle)]$. Two different paths, $(|e\rangle,|g\rangle)$ or $(|g\rangle,|e\rangle)$, lead to a component with zero phase. The atomic states, here, correspond to the cavity crossing. The Ramsey pulse $R_{2}$ mixes the states at the exit of $C$. The atomic detection in $D$ thus does not provide any information about the states in $C$ (a 'quantum eraser' situation). The two paths leading to the zero-phase component are indistinguishable, resulting in a quantum interference. This interference vanishes when decoherence has turned the cat superposition into a statistical mixture before $A_{p}$ enters $C$. Let us show now that this interference process reflects on an atomic signal $\eta$ :

$$
\eta=P_{g \mid g}-P_{e \mid g},
$$

where $P_{g \mid g}$ and $P_{e \mid g}$ are the conditional probabilities for detecting the mouse atom $A_{p}$ in $|g\rangle$ or $|e\rangle$, provided $A_{i}$ has been detected in $|g\rangle$.

Let us thus follow the successive state transformations, taking relaxation into account with the simple kitten model presented in the previous Section. When $A_{i}$ has been detected in $|g\rangle$, according to Eq.(74), the field has been prepared at $t=0$ in:

$$
\left|\Psi_{\Phi_{0}}^{+}\right\rangle=\frac{1}{\sqrt{2}}\left[\left|\beta e^{-i \Phi_{0}}\right\rangle+\left|\beta e^{i \Phi_{0}}\right\rangle\right] .
$$

We assume here that the decoherence time scale is much longer than the atom-field interaction time $t_{i}^{d}$. Decoherence is then negligible during $t_{i}^{d}$ and only takes place during the time interval $t$ between the $A_{i}$ and $A_{p}$. When $A_{p}$ enters the apparatus, the field state has been partially entangled with $E$, the cavity+environment state being:

$$
\left|\Psi^{C E}(t)\right\rangle=\frac{1}{\sqrt{2}}\left[\left|\beta e^{-i \Phi_{0}}\right\rangle \otimes\left|E^{-}(t)\right\rangle+\left|\beta e^{i \Phi_{0}}\right\rangle \otimes\left|E^{+}(t)\right\rangle\right],
$$




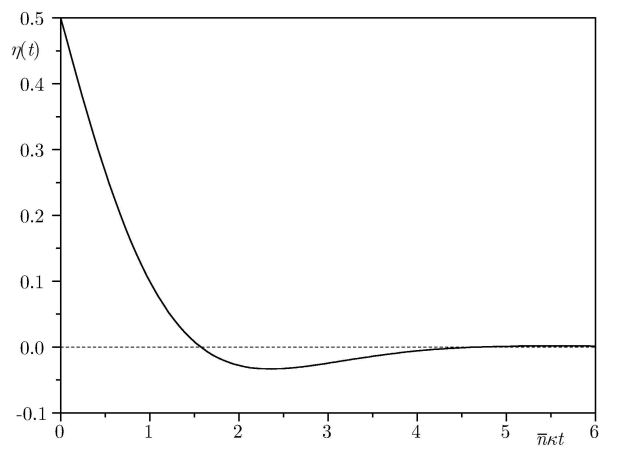

Figure 15: Theoretical value of the correlation signal $\eta(t)$ for $2 \Phi_{0}=\pi / 2$, as a function of $\kappa \bar{n} t$.

where $\left|E^{-}(t)\right\rangle$ and $\left|E^{+}(t)\right\rangle$ are the environment states defined by Eqs.(84) and (85). The probe atom $A_{p}$ then interacts with $C$. As $A_{i}$, it crosses $C$ in a time short compared to the decoherence time. The environment remains spectator during this interaction. Using the superposition principle, we can compute separately the effect of the $A_{p}-C$ interaction on the two parts of the cat state and add the resulting contributions:

$$
\begin{gathered}
\left|\Psi^{A_{i} E A_{p}}(t)\right\rangle=\frac{1}{2 \sqrt{2}}\left[e^{-i \Phi_{0}}\left|e_{2}\right\rangle \otimes\left(\left|\beta e^{-2 i \Phi_{0}}\right\rangle-|\beta\rangle\right)+\right. \\
\left.+\left|g_{2}\right\rangle \otimes\left(\left|\beta e^{-2 i \Phi_{0}}\right\rangle+|\beta\rangle\right)\right] \otimes\left|E^{-}(t)\right\rangle+ \\
+\frac{1}{2 \sqrt{2}}\left[e^{-i \Phi_{0}}\left|e_{2}\right\rangle \otimes\left(|\beta\rangle-\left|\beta e^{2 i \Phi_{0}}\right\rangle\right)+\right. \\
\left.+\left|g_{2}\right\rangle \otimes\left(|\beta\rangle+\left|\beta e^{2 i \Phi_{0}}\right\rangle\right)\right] \otimes\left|E^{+}(t)\right\rangle .
\end{gathered}
$$

We get the three phase components represented in figure 14. The first two and last two lines in the r.h.s. describe the 'offsprings' of the $\left|\beta e^{-i \Phi_{0}}\right\rangle$ and $\left|\beta e^{+i \Phi_{0}}\right\rangle$ components of the cat left by $A_{i}$ in $C$. Each one is tagged by a different environment state, carrying some information about its the phase. We get thus the probabilities $P_{g \mid g}$ and $P_{e \mid g}$ for detecting the second atom in $|g\rangle$ or $|e\rangle$ (after having found $A_{i}$ in $\left.|g\rangle\right)$ :

$$
\begin{aligned}
P_{g \mid g} & =\frac{1}{2}+\frac{1}{4} \operatorname{Re}\left\langle E^{-}(t) \mid E^{+}(t)\right\rangle ; \\
P_{g \mid g} & =\frac{1}{2}-\frac{1}{4} \operatorname{Re}\left\langle E^{-}(t) \mid E^{+}(t)\right\rangle .
\end{aligned}
$$

Replacing $\left\langle E^{-}(t) \mid E^{+}(t)\right\rangle$ by its expression (86), we obtain in the case $2 \Phi_{0} \neq \pi$ :

$$
\begin{aligned}
\eta(t) & =P_{g \mid g}-P_{e \mid g}=\frac{1}{2} \operatorname{Re} \exp \left[-\bar{n}\left(1-e^{-\kappa t}\right)\left(1-e^{2 i \Phi}\right)\right] \\
& =\frac{1}{2} e^{-2 \bar{n}\left(1-e^{-\kappa t}\right) \sin ^{2} \Phi_{0}} \cos \left[\bar{n}\left(1-e^{-\kappa t}\right) \sin \left(2 \Phi_{0}\right)\right] .
\end{aligned}
$$

For $\kappa t \ll 1$ this expression can be approximated by:

$$
\eta(t) \approx \frac{1}{2} e^{-2 \bar{n} \kappa t \sin ^{2} \Phi_{0}} \cos \left[\bar{n} \kappa t \sin \left(2 \Phi_{0}\right)\right] .
$$

The two-atom correlation signal varies from $1 / 2$ to 0 as decoherence proceeds. Its evolution is described by the product of a decaying exponential by a cosine function of time. The decay rate of the exponential, $1 / T_{D}=2 \kappa \bar{n} \sin ^{2} \Phi_{0}$, is the cavity damping rate $\kappa$ multiplied by the square of the distance in phase space $D^{2}=2 \bar{n} \sin ^{2} \Phi_{0}$ of the initial cat components. This is, once again, a characteristic feature of decoherence. The cosine factor is very close to 1 at the beginning of the 


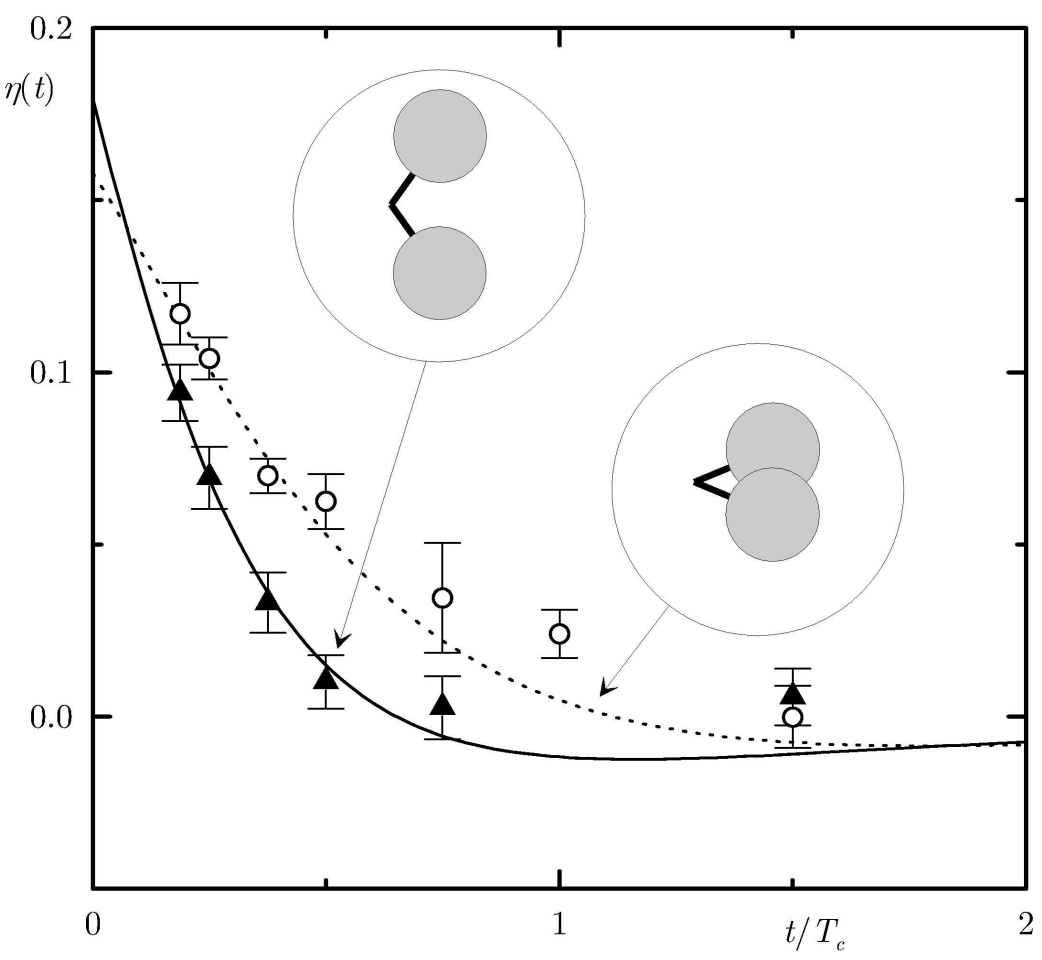

Figure 16: Two-atom correlation signal $\eta$ as a function of $t / T_{c}$ for an atom-cavity detuning $\Delta_{c} / 2 \pi=$ $170 \mathrm{kHz}$ (open circles) and $\Delta_{c} / 2 \pi=70 \mathrm{kHz}$ (solid triangles). The points are experimental, with statistical error bars. The curves present the theoretical signal, scaled by a factor taking into account experimental imperfections. The cat phase components are pictorially depicted in the insets.

evolution. When $\eta(t)$ is already strongly reduced, this factor can change sign, adding a modulation in the tail of the $\eta(t)$ function. Figure 15 represents the theoretical variation of $\eta(t)$ for $2 \Phi_{0} \sim \pi / 2$. Note that the maximum $\eta$ value is twice larger (1) when $\Phi_{0}=\pi / 2$, because then the two states $\left|\beta e^{-2 i \Phi_{0}}\right\rangle$ and $\left|\beta e^{+2 i \Phi_{0}}\right\rangle$ coincide. This gives rise to another interference effect in the $P_{g \mid g}$ and $P_{e \mid g}$ probabilities.

Sending pairs of atoms across the Ramsey interferometer, we have observed the evolution of $\eta$ [7]. The cavity damping time in this early experiment was $160 \mu \mathrm{s}$, making the decoherence fast and limiting the experiment to small values of $\bar{n}$. The cat state prepared by the first atom contained $\bar{n}=3.3$ or 5.1 photon on average. The phase splitting $2 \Phi_{0}$ was set at two values (100 and 50 degrees) by choosing the detuning $\Delta_{c}(70 \mathrm{kHz}$ and $170 \mathrm{kHz}$ respectively). The separation $t$ between the two atoms was varied between $30 \mu \mathrm{s}$ and $250 \mu \mathrm{s}$.

Figure 16 shows $\eta(t)$ for $\bar{n}=3.3$ and two splitting angles. The points are experimental and the lines theoretical. Note that the theory is not restricted to the simple perturbative analysis developed above. It includes higher order terms in $\Omega_{0} / \Delta_{c}$ correcting the expression of the cat states at $\Delta_{c}=70 \mathrm{kHz}$. We have also incorporated the finite Ramsey fringes contrast, which explains why the maximum correlation is only 0.18 .

The correlation signals decrease with $t$, revealing directly the dynamics of decoherence. The agreement with the theoretical model is excellent. Most strikingly, decoherence proceeds at a faster rate when the distance between cat state components increases. A decoherence time $\approx 0.24 / \kappa$, much shorter than the photon decay time, is found for the larger cat $\left(\Delta_{c}=70 \mathrm{kHz}\right)$. A similar agreement with theory is obtained when comparing, for the same $\Delta_{c}=70 \mathrm{kHz}, \eta(t)$ for different $\bar{n}$ values $(5.1$ and 3.3).

The CQED cat experiments are, as discussed above, models of an ideal quantum measurement. The coherent field is a meter measuring the state of the first atom crossing the cavity and evolving 
under the effect of their interaction into a mesoscopic superposition. This decoherence experiment illustrates vividly the fast evolution of the atom + meter state towards a statistical mixture and the increasing difficulty to maintain quantum coherence when the distance between the components of the mesoscopic superposition is increased.

\section{The future of cavity cats}

We have presented above a first experiment on decoherence dynamics. It would be quite interesting to dive deeper into the quantum/classical transition and to test our understanding of decoherence in this textbook situation. It is thus essential to get more information on the relaxing cat state than provided by the correlation $\eta$. Finally, it would be interesting to merge two of the most intriguing features of the quantum world: decoherence and non-locality. We address these issues here. We show first that the resonant atom-field interaction can produce efficiently very large cat states. We present a direct measurement of the cavity field Wigner function. Finally, we discuss an experiment under construction aiming at the preparation of non-local Schrödinger cat states.

\subsection{Generation of large cats by resonant atom-field interaction}

In the preceding Section, we learned how to generate Schrödinger cats via the dispersive atomcavity interaction, valid when the atom-cavity detuning is large and the photon number small. It is tempting to increase the photon number and to decrease $\Delta_{c}$ in order to generate 'larger' cats. What happens then when $\Delta_{c}$ goes all the way to zero? What is the index of refraction of a single resonant atom? In the classical Lorentz model of harmonically bound electrons [36], the answer is simple: the index is one. When it comes to a mesoscopic field, the situation is more interesting. The atom is indeed in a quantum superposition of two very large indices, resulting in the efficient generation of a Schrödinger cat state.

\subsubsection{Quantum Rabi oscillations in a mesoscopic field}

We thus return to the interaction of a single atom, initially in $|e\rangle$, with a coherent state $|\alpha\rangle=$ $\sum_{n} c_{n}|n\rangle$ containing $\bar{n}=|\alpha|^{2}$ photons on the average. The initial atom-cavity state, $|e\rangle \otimes|\alpha\rangle$, can be expanded on the dressed atom state basis $| \pm, n\rangle$ and the evolution easily computed. The state at the effective interaction time $t_{i}$ is:

$$
|\Psi\rangle=\frac{1}{\sqrt{2}}\left[\left|\Psi_{1}\right\rangle+\left|\Psi_{2}\right\rangle\right]
$$

where

$$
\left|\Psi_{1}\right\rangle=\frac{1}{\sqrt{2}}\left[\sum_{n} c_{n} e^{i \Omega_{0} \sqrt{n+1} t_{i} / 2}(|e, n\rangle-i|g, n+1\rangle)\right]
$$

and

$$
\left|\Psi_{2}\right\rangle=\frac{1}{\sqrt{2}}\left[\sum_{n} c_{n} e^{-i \Omega_{0} \sqrt{n+1} t_{i} / 2}(|e, n\rangle+i|g, n+1\rangle)\right] .
$$

This is an exact expression. The probability $P_{e}(t)$ for finding the atom in $|e\rangle$ is simply:

$$
P_{e}(t)=\sum_{n}\left|c_{n}\right|^{2} \frac{1+\cos \Omega_{0} \sqrt{n+1} t_{i}}{2}
$$

a sum of terms oscillating at the frequencies $\Omega_{0} \sqrt{n+1}$, weighted by the probability for getting the corresponding photon number $n$ in the initial coherent cavity state.

Figure 17 shows $P_{e}(t)$ for $\bar{n}=15$. This Rabi oscillation signal presents remarkable features, which attracted a lot of interest in the early days of quantum optics [37, 38]. At short times, when $\bar{n} \gg 1$, an oscillation is observed at a frequency $\Omega_{0} \sqrt{\bar{n}+1}$, proportional to the classical field amplitude. This oscillations is quite rapidly damped away and $P_{e}$ reaches a stationary $1 / 2$ 


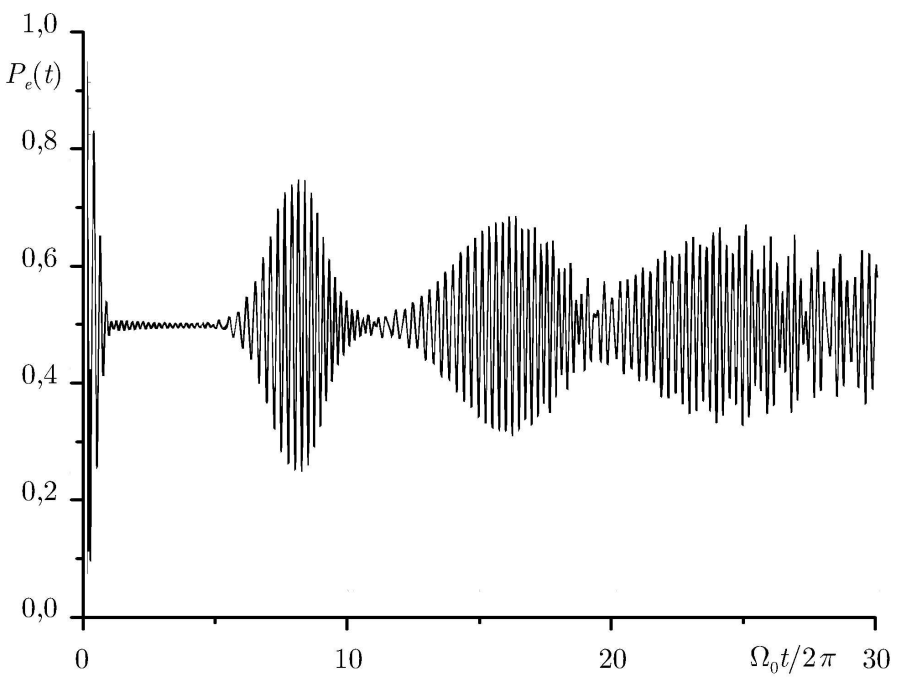

Figure 17: Quantum revivals: computed probability $P_{e}(t)$ for finding the atom in state $|e\rangle$ versus the interaction time $t$ in units of $2 \pi / \Omega_{0}$. The cavity contains initially an $\bar{n}=15$ photons coherent field.

value. This 'collapse' is due to the dispersion of Rabi frequencies in Eq.(99). At much longer times, however, the oscillations 'revive', due to the rephasing of the finite set of oscillating terms in Eq.(99). This revival is directly linked to the quantization of the Rabi oscillation spectrum and, hence, to the field energy quantization itself. This explains the theoretical interest devoted to this phenomenon [39, 40, 41, 42]. We give below an enlightening interpretation of the collapse and revival in terms of complementarity.

The expression (96) is explicit, but does not provide a physical insight into the field evolution. We proceed thus to obtain a more explicit, if approximate, expression of the atom-cavity state, following [40]. We separate the kets $\left|\Psi_{1}\right\rangle$ and $\left|\Psi_{2}\right\rangle$ into an atom and a field part. With a mere redefinition of the running index $n$ :

$$
\left|\Psi_{1}\right\rangle=\frac{1}{\sqrt{2}}\left[\sum_{n} c_{n} e^{i \Omega_{0} \sqrt{n+1} t_{i} / 2}|e, n\rangle-i \sum_{n} c_{n-1} e^{i \Omega_{0} \sqrt{n} t_{i} / 2}|g, n\rangle\right],
$$

still an exact expression. We now assume that $\bar{n}$ and the photon number variance $\Delta N=\sqrt{\bar{n}}$ are much larger than 1 . The $c_{n}$ amplitudes are slowly varying functions of $n$ and we can set $c_{n} \approx c_{n-1}$. We use, in the exponentials, a first order expansion of $\sqrt{n+1}$ :

$$
\frac{\Omega_{0} \sqrt{n+1} t_{i}}{2} \approx \frac{\Omega_{0} \sqrt{n} t_{i}}{2}+\frac{\Omega_{0} t_{i}}{4 \sqrt{n}},
$$

which leads to:

$$
\left|\Psi_{1}\right\rangle=\frac{1}{\sqrt{2}}\left[\sum_{n} c_{n} e^{i \Omega_{0} \sqrt{n} t_{i} / 2}|n\rangle\right]\left[e^{i \Omega_{0} t_{i} / 4 \sqrt{\bar{n}}}|e\rangle-i|g\rangle\right],
$$

a product of independent atom and field states.

We then expand the $\sqrt{n}$ term around $n=\bar{n}$, up to the first order:

$$
\sqrt{n}=\frac{\sqrt{\bar{n}}}{2}+\frac{n}{2 \sqrt{\bar{n}}} .
$$

This is legitimate since $\Delta N \ll \bar{n}$. We get:

$$
\left|\Psi_{1}\right\rangle=\frac{1}{\sqrt{2}} e^{i \Omega_{0} \sqrt{\bar{n}} t_{i} / 4}\left[\sum_{n} c_{n} e^{i n \Omega_{0} t_{i} / 4 \sqrt{\bar{n}}}|n\rangle\right] \otimes
$$




$$
\otimes\left[e^{i \Omega_{0} t_{i} / 4 \sqrt{\bar{n}}}|e\rangle-i|g\rangle\right]
$$

The field state reduces thus to:

$$
\sum_{n} c_{n} e^{i n \Omega_{0} t_{i} / 4 \sqrt{\bar{n}}}|n\rangle=\left|\beta e^{i \Omega_{0} t_{i} / 4 \sqrt{\bar{n}}}\right\rangle
$$

a coherent state obtained from the initial one by a phase shift $\Omega_{0} t_{i} / 4 \sqrt{\bar{n}}$.

A similar calculation is performed for $\left|\Psi_{2}\right\rangle$. Grouping all the terms, we finally obtain the atom-field wavefunction as:

$$
\left|\Psi\left(t_{i}\right)\right\rangle \approx \frac{1}{\sqrt{2}}\left[\left|\Psi_{a}^{+}\left(t_{i}\right)\right\rangle \otimes\left|\Psi_{f}^{+}\left(t_{i}\right)\right\rangle+\left|\Psi_{a}^{-}\left(t_{i}\right)\right\rangle \otimes\left|\Psi_{f}^{-}\left(t_{i}\right)\right\rangle\right],
$$

where the atom wavefunctions write:

$$
\left|\Psi_{a}^{ \pm}\right\rangle=\frac{1}{\sqrt{2}} e^{ \pm i \Omega_{0} \sqrt{\bar{n}} t_{i} / 2}\left[e^{ \pm i \Omega_{0} t_{i} / 4 \sqrt{\bar{n}}}|e\rangle \mp i|g\rangle\right]
$$

and the field ones:

$$
\left|\Psi_{f}^{ \pm}\right\rangle=e^{\mp i \Omega_{0} \sqrt{\bar{n}} t_{i} / 4}\left|\beta e^{ \pm i \Omega_{0} t_{i} / 4 \sqrt{\bar{n}}}\right\rangle .
$$

Equation (106) describes a quantum superposition involving two coherent states rotating slowly, at an angular frequency $\Omega_{0} / 4 \sqrt{\bar{n}}$, in opposite directions in phase space, away from the initial state $|\alpha\rangle$. These components are correlated to atomic states, superpositions of $|e\rangle$ and $|g\rangle$ with equal weights, also rotating slowly in opposite directions in the equatorial plane of the Bloch sphere, so that the phase relationship between the atomic dipole and the associated field components are kept throughout the evolution.

In other words, a resonant atom, initially in state $|e\rangle$, is in a quantum superposition of two opposite indices of refraction and leads to a cat state preparation. The components angular frequencies, $\pm \Omega_{0} / 4 \sqrt{\bar{n}}$, go to zero in the classical limit of a very large field (obtained by letting $\bar{n} \rightarrow \infty$ and $\Omega_{0} \rightarrow 0$ so that the Rabi frequency $\Omega_{0} \sqrt{\bar{n}}$ remains constant). This phase separation is thus a mesoscopic feature, directly related to the photon graininess. Its velocity is larger than for any finite atom-cavity detuning, much faster than in the dispersive regime. The resonant method is thus optimal for the preparation of photonic cats with many photons.

We have so far overlooked the global phase factors multiplying the expressions of the $\left|\Psi_{a}^{ \pm}\right\rangle$ and $\left|\Psi_{f}^{ \pm}\right\rangle$states. They evolve at frequencies about $\bar{n}$ times larger than the phase drift frequency. In the $\left(\left|\Psi_{1}\right\rangle+\left|\Psi_{2}\right\rangle\right) / \sqrt{2}$ superposition, though, their role is essential. They give rise to the interference effect responsible for the Rabi oscillation. The study of the photonic cat evolution is thus intimately related to the dynamics of the precession between $|e\rangle$ and $|g\rangle$.

This leads to a simple interpretation of the quantum Rabi collapse and revivals, again in terms of complementarity. At short times, the two coherent components overlap and the quantum interference and hence the Rabi oscillations show up. Soon, the two coherent components separate. The cavity field acts then as a which-path detector for the interfering atomic states, and the Rabi oscillations disappear. The evolution does not stop, though, since the slow rotation of the coherent states in phase space goes on. After a time $t_{r}$ such that $t_{r} \Omega_{0} / 4 \sqrt{\bar{n}}=\pi$, the coherent states have undergone a $\pi$ rotation in opposite directions. They again overlap. The which-path information is erased and the atom-field entanglement is broken. The Rabi oscillations revive. A series of revivals is thus expected, corresponding to the periodic overlaps of the counter-rotating fields. That these revivals get progressively broadened, with a lower than unity contrast, as conspicuous on figure 17 , is due to higher order terms neglected in our approximations.

\subsubsection{Observing the field phase separation}

We have evidenced the resonant phase separation via a measurement of the final field phase distribution [43]. The experiment is similar to the measurement of the dispersive phase shifts described above. It involves two successive field injections and two atoms. A first coherent state $|\alpha\rangle$ is prepared, with $\bar{n}$ between 10 and 40. A resonant atom $A_{r}$ is sent across the cavity at a fixed velocity 


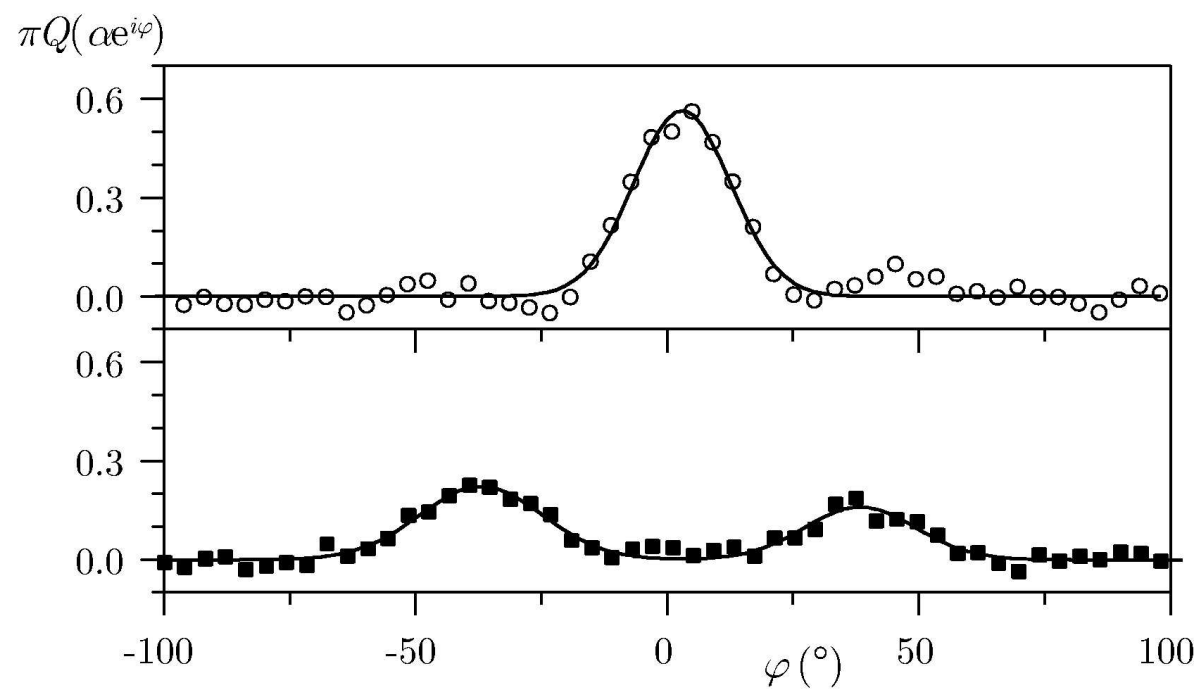

Figure 18: Phase distribution $Q\left(\alpha e^{i \varphi}\right)$ (in units of $1 / \pi$ ) for the resonant cat state. Top curve: initial coherent field, with 29 photons on the average. Bottom: phase distribution at $t_{i}=52 \mu \mathrm{s}$. The points are experimental and the solid curves are Gaussian fits.

$v_{1}=335 \mathrm{~m} / \mathrm{s}$ or $v_{2}=200 \mathrm{~m} / \mathrm{s}$ (corresponding to a total effective interaction time $t_{i}=32 \mu \mathrm{s}$ or $52 \mu \mathrm{s})$.

A second coherent field $|\beta\rangle=\left|-\alpha e^{i \varphi}\right\rangle$, with the same amplitude as the first, but a variable phase $\varphi$, is then added in $C$. A probe atom, $A_{p}$, also resonant with $C$, follows. Its absorption yields as above the $Q$ function of the field left in the cavity by $A_{r}$.

Figure 18 presents $Q\left(\alpha e^{i \varphi}\right)$ versus $\varphi$ for $\bar{n}=29$ and $t_{i}=52 \mu \mathrm{s}$. As expected, a double peak distribution is obtained, with a phase splitting of 1.3 radians, in good agreement with the theoretical value $\Omega_{0} t_{i} / 2 \sqrt{\bar{n}}=1.5$ radians. We have checked that the splitting is proportional to $t_{i}$ and inversely proportional to $\alpha$. The observed phase shifts are in good agreement with the simple first order calculation presented above and in excellent agreement with a numerical simulation of the exact atom-cavity interaction [43]. The maximum phase splitting, for $\bar{n}=15$ and $t_{i}=52 \mu \mathrm{s}$, was $90^{\circ}$, a phase space separation much larger than what can be obtained in the dispersive regime.

\subsubsection{Checking the cat coherence}

The $Q$ function measurement does not provide any hint about the coherent nature of the superposition. The simplest coherence assessment would be the spontaneous Rabi revival observation. Unfortunately, even for a relatively small cat with $\bar{n}=13$, the revival time $t_{r}=4 \pi \sqrt{\bar{n}} / \Omega_{0}=260 \mu \mathrm{s}$ is prohibitively long.

It is possible to bypass this difficulty and to force the system to undergo an early revival. The trick is to let the field components separate in phase space until a time $T$, shorter than $t_{r} / 2$, then to force them to come back on their tracks and to recombine at the initial phase. The recombination time, $2 T$, can then be shorter that $t_{r}$. This method, reminiscent of the spin echoes used in NMR, has been proposed by Morigi et al. [44].

The system's evolution is ruled by the Jaynes Cummings Hamiltonian, given at resonance and cavity center by $H_{a c}$ [see Eq.(54)]. We time the evolution with a 'clock' synchronized on the effective interaction time $t_{i}$. The experiment can be described as if the atom was sitting at cavity center. In an echo sequence, the system evolution from time 0 to $T$ is described by the evolution operator $U_{1}=\exp \left(-i H_{a c} T / \hbar\right)$. The atom undergoes, at time $T$, a percussional phase kick corresponding to the unitary operation $\sigma_{Z}$. This kick can be produced by an electric field pulse, transiently shifting the atomic levels via Stark effect. This pulse phase shifts by $\pi$ the atomic dipole states, reversing their phase relationship with the associated coherent component. The evolution of the coupled 
atom-field states is then reversed, both in the Fresnel plane and in the equatorial plane of the Bloch sphere.

More quantitatively, after the phase shifting pulse, the Jaynes-Cummings evolution resumes for the remaining time $t_{i}-T$, with the evolution operator $U_{2}=\exp \left[-i H_{a c}\left(t_{i}-T\right) / \hbar\right]$. The overall evolution operator is:

$$
U=U_{2} \sigma_{Z} U_{1}=\sigma_{Z}^{2} U_{2} \sigma_{Z} U_{1}=\sigma_{Z} e^{-i H_{a c}\left(2 T-t_{i}\right) / \hbar}
$$

where we have used the identity $\sigma_{Z}^{2}=1$ and:

$$
\sigma_{Z} H_{a c} \sigma_{Z}=-H_{a c}
$$

Eq.(110) means that the evolution after the phase kick is the time-mirror image of the evolution between 0 and $T$. At time $t_{i}=2 T$, the evolution brings the system back to its initial state, up to a global $\pi$-phase shift between the amplitudes associated to levels $|e\rangle$ and $|g\rangle$. Provided the whole evolution has been coherent, Rabi oscillations, exactly identical to the initial ones, show up around $2 T$.

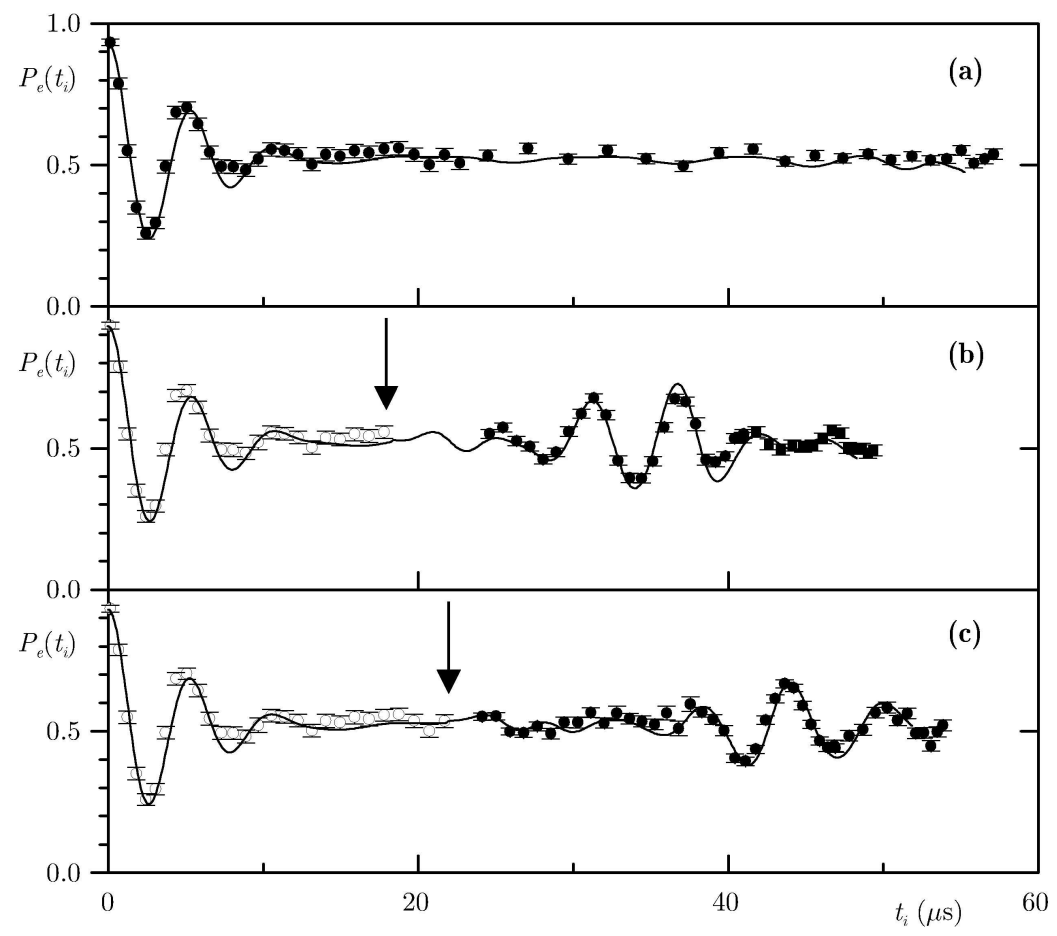

Figure 19: Experimental echo signals. Probability $P_{e}$ for detecting the atom in $|e\rangle$ as a function of the atom-cavity effective interaction time $t_{i}$. The points are experimental with statistical error bars and the solid lines are a theoretical fit. (a) Rabi collapse without Stark pulse. (b) and (c) Stark pulse applied at 18 and $22 \mu$ s respectively (vertical arrows). The first part of the signal (open circles) is reproduced from (a) for visual convenience.

We have applied this time reversal method to the study of the Rabi oscillation in our CQED set-up [45]. The atomic velocity is set at $v=156 \mathrm{~m} / \mathrm{s}$ and $\bar{n}=13.6$. The atom is tuned into resonance with $C$ at $t_{i}=0$. The Rabi oscillation starts and collapses while the atom is still near cavity entrance. In a preliminary experiment, this signal is recorded by freezing the evolution at increasing times. The final atomic state is recorded and we reconstruct the probability $P_{e}\left(t_{i}\right)$ for finding the atom in level $|e\rangle$. The Rabi collapse signal is shown in Figure 19(a).

We then perform the echo experiment. The Stark phase kicking pulse is applied to the atom when it reaches cavity center, long after the Rabi collapse is complete. The atomic frequency then 
resumes its resonant value and the atomic evolution proceeds until a final time $t_{i}=t_{f}$, at which the atom is suddenly detuned. The frozen atomic state is analyzed by the atomic detector. Traces 19(b) and (c) show the signals obtained when the kicking pulse occurs at the effective time $t_{i}=T=18 \mu \mathrm{s}$ and $22 \mu$ s respectively. The echo signals around time $2 T$ are clearly observed, with a contrast about $50 \%$ of the ideal value. The kicking time $T=22 \mu$ s corresponds to 2.5 damping times of the initial Rabi signal. The maximum separation of the field components reaches 0.90 radians, about three times the phase fluctuation of the initial coherent field $(1 / \sqrt{\bar{n}}=0.27$ radian). The components of the field are thus well separated, before being recombined by the time reversal operation. This echo signal reveals the existence of a mesoscopic coherence in the atom-field system between the collapse and the induced revival time.

The induced revival signal could, in principle, be used to assess the decoherence process. The observed echo contrast should be directly related to the decoherence integrated from $t_{i}=0$ to $t_{i}=2 T$. In practice, this analysis cannot be carried out because the echo is affected by experimental imperfections. A numerical simulation of the system's evolution (including the effect of decoherence due field damping in the cavity and all known causes of imperfections), shown as solid lines in figure 19 , indicates that the contrast reduction is dominated by mundane imperfections. Decoherence would take over for longer echo times, whose observation requires the use of slower atoms, not readily available.

\subsubsection{How big a cat?}

What is the maximal practical size of a cat in these cavity QED experiments? The longest cavity damping time obtained in 2005, is $T_{c}=14 \mathrm{~ms}$, a two-order of magnitude increase with respect to the 1996 experiment [7], opening the way to much fatter photonic cats. The limit to the cat size can be evaluated by comparing the time required to prepare and probe it to its decoherence time. With the optimal resonant coupling, the effective interaction time $t_{i}$ needs to be such that $\Omega_{0} t_{i} / 2 \sqrt{\bar{n}} \sim \pi$, i.e. $t_{i} \sim\left(2 \pi / \Omega_{0}\right) \sqrt{\bar{n}}$. It should be shorter than the decoherence time $1 / 2 \kappa \bar{n}=T_{c} / 2 \bar{n}$, thus:

$$
\bar{n}<\left(\frac{\Omega_{0} T_{c}}{2 \pi}\right)^{2 / 3}=\left(\frac{\Omega_{0} Q}{2 \pi \omega}\right)^{2 / 3} .
$$

An increase in $T_{c}$ and $Q$ by two orders of magnitude corresponds to a $10^{2 / 3} \approx 20$-fold improvement over the limit $(\bar{n} \approx 5)$ of the early cat experiments. Cat states with $\bar{n}$ as large as 100 are thus in view.

The $Q$ factor is still limited by mirror imperfections. With ideally smooth mirrors, and low enough temperature we could reach the diffraction limit, when the losses occur by photon scattering at the edge of the mirrors. With the current Fabry-Perot geometry, this would correspond to $Q \approx 4.10^{11}$, or $T_{c}=1.4 \mathrm{~s}$, again a two orders of magnitude improvement. We can dream of another 20-fold increase in the cat size up to 2000 photons. This limit would be difficult to break, but it leaves a lot of room for decoherence experiments.

\subsection{Imaging Schrödinger cats: a direct determination of the cavity field Wigner function}

The smoking guns of cat coherence obtained so far do not provide a full insight into the cavity state. It would be much more interesting to reconstruct the field's Wigner function $W$, which provides a full information about the cat and its coherence. We made a first step in this direction by measuring the Wigner distribution of a non-classical cavity field, here an approximate single-photon state [33].

The method was proposed by Lutterbach and Davidovich [46]. Let us recall Eq. $(40): W(\alpha)$ is the average of the parity operator $\mathcal{P}$ in the field translated by $-\alpha$. This translation, action on $C$ of a displacement operator $D_{1}$, is easily produced by the source $S$. To measure $\langle P\rangle$ [33], we send across $C$ a probe atom $A_{p}$, initially in $|g\rangle$, tuned off-resonance from $C$. The $|e\rangle \rightarrow|g\rangle$ transition is transiently light-shifted by the cavity field, resulting in a phase shift of the atomic coherence. We adjust the atom-cavity detuning, $\delta=2 \pi \times 105 \mathrm{kHz}$, and the atomic velocity, $v=154 \mathrm{~m} / \mathrm{s}$, so that a single photon produces a $\pi$-phase shift on the $|e\rangle /|g\rangle$ coherence. 
This phase shift is revealed by the Ramsey interferometer sandwiching $C$. The probability $P_{e}$ for detecting $A_{p}$ in $|e\rangle$ is modulated versus the relative phase $\phi$ of the $R_{1}$ and $R_{2}$ Ramsey pulses resonant with the $|e\rangle \rightarrow|g\rangle$ transition: $P_{e}=(1+\cos \phi) / 2$. An $n$-photon field in $C$ shifts the interference pattern by $n \pi$. When the photon distribution is $p_{n}$ after $D_{1}, P_{e}$ becomes $P_{e}(\phi)=$ $\left[1+\sum_{n}(-1)^{n} p_{n} \cos \phi\right] / 2=[1+\langle P\rangle \cos \phi] / 2$. Hence, $W$ is directly related to the Ramsey fringes contrast $c(\alpha)$ :

$$
W=\frac{2}{\pi}\langle P\rangle=\frac{2}{\pi} c(\alpha)=\frac{2}{\pi}\left[P_{e}(0, \alpha)-P_{e}(\pi, \alpha)\right]
$$
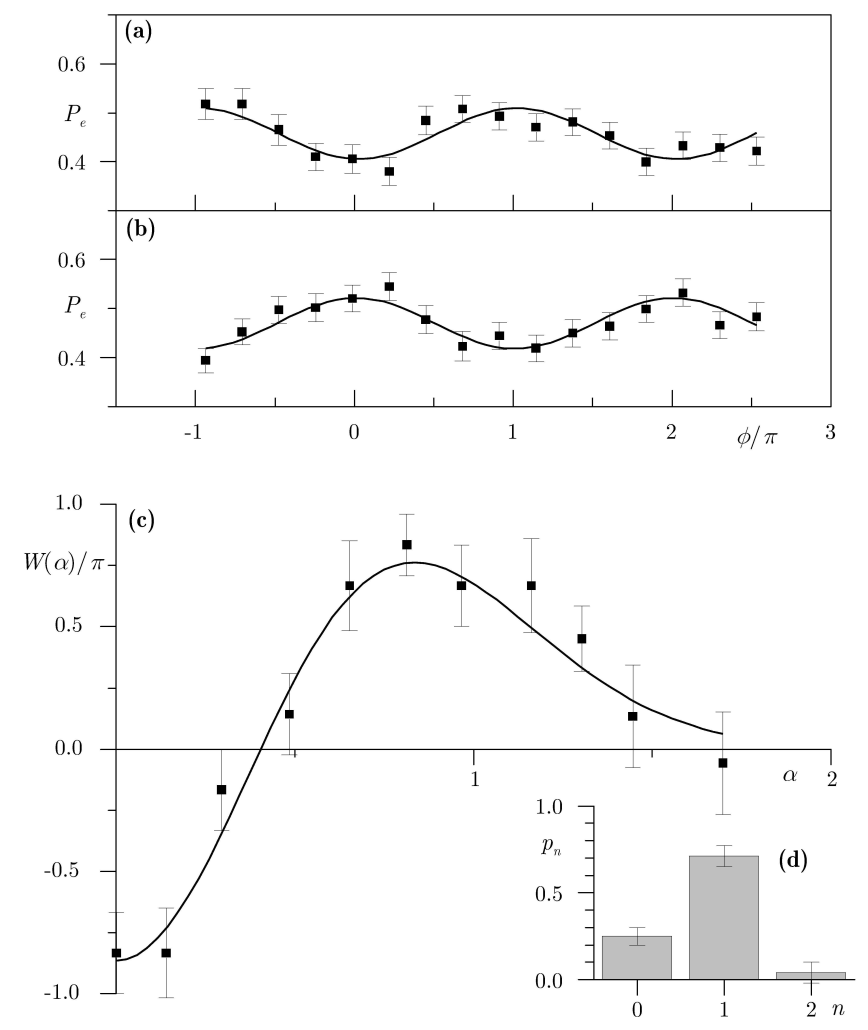

Figure 20: Determination of the 'one-photon' Wigner function. (a) Ramsey fringes for $\alpha=0$. Probability $P_{e}$ for detecting the atom in state $|e\rangle$ as a function of the Ramsey interferometer phase $\phi / \pi$. Dots are experimental with statistical error bars. The solid curve is a sine fit. (b) Ramsey fringes for $\alpha=0.81$. (c) Dots: $W(\alpha)$ with error bars reflecting the uncertainty on the Ramsey fringes fit. The solid line is a theoretical fit. (d) Corresponding photon number distribution $p_{n}$.

We have applied this procedure to the residual thermal field in the cavity and to an approximate single-photon Fock state, prepared through an atomic emission in $C$. The latter results are shown in figure 20. Panels (a) and (b) present the Ramsey fringes for two displacement amplitudes $\alpha=0$ and $\alpha=0.81$ ( $W$ being phase-independent, we need not tune the phase of $D_{1}$ and can assume that $\alpha$ is real). The fringe phase is shifted by $\pi$ between these two values, indicating a sign change for $W$, from negative ( $\operatorname{small} \alpha$ ) to positive (large $\alpha$ )

The fringes are fitted with sine curves [solid lines in fig. 20(a)-(b)], providing the contrast $c(\alpha)$ $( \pm 0.02$ uncertainty). Figure 20(c) presents the Wigner distribution values deduced from these fits (dots). The measurements are affected by the finite intrinsic contrast of the Ramsey interferometer. We thus multiply the raw data by a 4.16 factor, in order to obtain a normalized Wigner function. The measured $W$ function is negative around the origin, revealing the non-classical nature of the cavity state. The solid line in Fig. 20 (c) is a fit on a mixture of Fock states with an adjustable 
photon distribution, $p_{n}$. The inferred $p_{n}$ is shown in Fig. 20(d). It exhibits a $71 \%$ probability for the one-photon state. This value is in agreement with a model of experimental imperfections taking cavity relaxation into account.

This measurement opens interesting perspectives for the monitoring of the cat relaxation. It should be possible, in the near future, to reconstruct experimentally the evolution of the cat's Wigner function shown in figure 13.

\subsection{Towards non-local cats}

The Wigner function measurement can be used for the investigation of a new type of quantum field, merging two intriguing aspects of quantum mechanics: a non-local Schrödinger cat state. This state is a mesoscopic quantum superposition, exhibiting a fast decoherence as the cats encountered so far. At the same time, it exhibits the non-local properties of an EPR pair of particles and could lead to the observation of a violation of Bell inequalities [47]. This violation should be rapidly washed out by decoherence process.

We have in mind an experiment with two cavities, $C_{1}$ and $C_{2}$, tuned slightly off-resonance with the $|g\rangle \rightarrow|e\rangle$ transition, initially prepared in the same coherent state $|\gamma\rangle$ by two classical sources $S_{1}$ and $S_{2}$. An atom is prepared, in a first Ramsey zone $R_{1}$ before $C_{1}$, in a coherent superposition $(|g\rangle+|i\rangle) / \sqrt{2}$, where $|i\rangle$ is the circular state with principal quantum number 49 . When in $|i\rangle$, the atom is far off-resonance and leaves the cavity fields unchanged. When in $|g\rangle$, the atom produces instead a $\pi$-phase shift of both fields. A second Ramsey zone, $R_{2}$, after $C_{2}$ mixes again $|g\rangle$ and $|i\rangle$, erasing any information about the atomic state in the cavities. The atomic detection in $|g\rangle$ then projects the two cavities into:

$$
|\Psi\rangle=\frac{1}{\sqrt{N}}(|\gamma, \gamma\rangle+|-\gamma,-\gamma\rangle)
$$

where $N$ is a normalization factor, close to 2 for large $\alpha$ values. This is a mesoscopic quantum superposition and also a non-local pair, since the two cavity field amplitudes exhibit quantum correlations.

In EPR experiments with spin pairs [48, 49], the Bell inequalities involve averages of products of binary spin observables $(-1$ or +1$)$ for four settings of the detection axes. In the two-field mode case, the spin observables are replaced by the photon number parity $\mathcal{P}$, another binary observable. Independently adjustable displacements in both cavities play the role of the detection axes settings. The Bell signal, $S_{b}$, involves then four values of the joint cavities four-dimensional Wigner function $W\left(\alpha_{1}, \alpha_{2}\right)[47]$ :

$$
S_{b}=\frac{\pi^{2}}{4}\left|W\left(\alpha^{\prime}, \beta^{\prime}\right)+W\left(\alpha, \beta^{\prime}\right)+W\left(\alpha^{\prime}, \beta\right)-W(\alpha, \beta)\right| .
$$

It should be lower than 2 for any local realistic description. It can reach $2 \sqrt{2}$ for large $\alpha$ values. It is already 2.6 for a two-photon cat $(\gamma=\sqrt{2})$.

The joint Wigner function can be determined as for a single cavity, with independent displacements of the cavity fields performed by $S_{1}$ and $S_{2}$ followed by a probe atom prepared in $R_{1}$ in a superposition of $|e\rangle$ and $|g\rangle$, whose coherence undergoes two $\pi$-phase shifts per photon in both cavities. The accumulated phase shift is probed in $R_{2}$. The contrast of the Ramsey fringes is then directly proportional to $W$. We have numerically simulated this experiment for two cavity damping times, $T_{c}=30 \mathrm{~ms}$ and $300 \mathrm{~ms}$, taking into account the exact atom-field interaction [50]. The results are presented in figure 21 as a function of the delay $T$ between state preparation and measurement. The Bell signal is above the classical limit for a short time interval and undergoes a fast decoherence.

This experiment puts severe requirement on the cavity quality, since the decoherence of the Bell signal is pretty fast. However, as mentioned above, we have recently obtained $T_{c}=14 \mathrm{~ms}$, already such that a violation of a Bell inequality could be observed in a short time interval for small photon numbers. This is certainly a strong incentive to build a two-cavity experiment. 


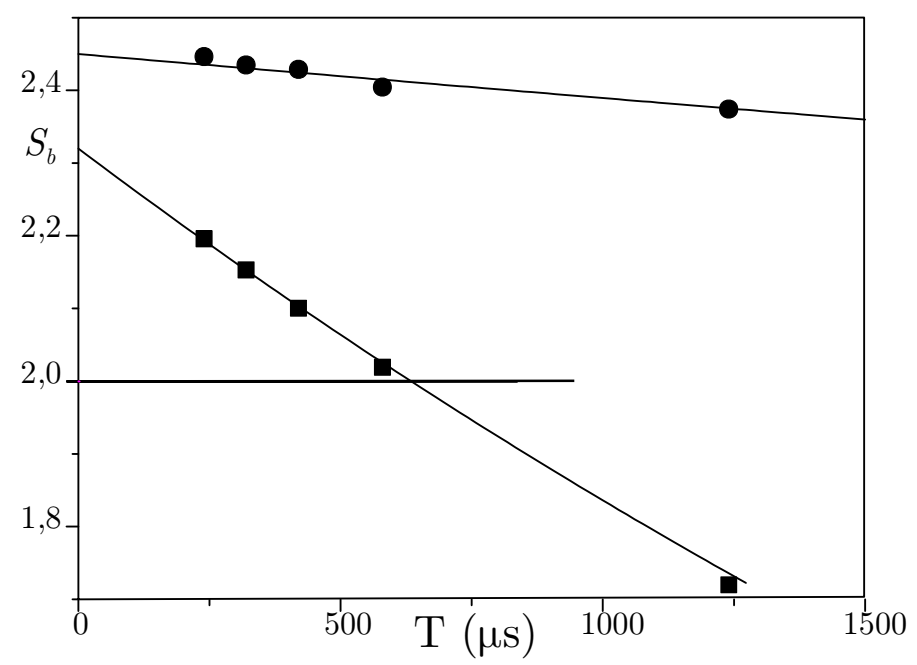

Figure 21: Bell signal $S_{b}$ as a function of the time delay $T$ between state preparation and analysis. The dots correspond to experimental simulations with $T_{c}=30 \mathrm{~ms}$ (squares) and $300 \mathrm{~ms}$ (circles). The lines are linear fits.

\section{Conclusion}

Cavity QED experiments with circular Rydberg atoms and superconducting cavities are well adapted to the experimental study of the decoherence dynamics. The interaction of a single atom with a mesoscopic field, either in the resonant or dispersive regimes, produces quantum superpositions of coherent components with different classical phases, mesoscopic versions of the famous Schrödinger cat state. The decoherence transforms these superpositions into statistical mixtures in a time much shorter than the cavity energy lifetime. Probing the field quantum state with another atom makes it possible to unveil directly the decoherence process.

There is clearly much to do after the first qualitative measurements presented here. The development of new techniques to image the cavity state, such as the direct determination of the cavity field Wigner function, the realization of cavities with a much higher quality factors, open the way to detailed, 'metrologic' studies of the decoherence of pretty large Schrödinger cats.

A new experimental set-up is presently in construction in our laboratory. It should circumvent many of the limitations of the present apparatus. It will incorporate two separate cavities, making it possible to prepare and probe the non-local cats described above. Besides these fundamental aspects, it will also be well suited for the realization of complex quantum information manipulations $[4]$.

\section{References}

[1] H. D. Zeh, Found. Phys. 1, 69 (1970).

[2] W. H. Zurek, Decoherence and the transition from quantum to classical, Phys. Today 44, 36 (octobre 1991).

[3] W. H. Zurek, Decoherence, einselection, and the quantum origins of the classical, Rev. Mod. Phys. 75, 715 (2003).

[4] J.-M. Raimond, M. Brune, and S. Haroche, Manipulating quantum entanglement with atoms and photons in a cavity, Rev. Mod. Phys. 73, 565 (2001).

[5] D. Leibfried, R. Blatt, C. Monroe, and D. J. Wineland, Quantum dynamics of single trapped ions, Rev. Mod. Phys. 75, 281 (2003). 
[6] C. Monroe, D. M. Meekhof, B. E. King, and D. J. Wineland, A "Schrödinger cat" superposition state of an atom, Science 272, 1131 (1996).

[7] M. Brune, E. Hagley, J. Dreyer, X. Maître, A. Maali, C. Wunderlich, J.-M. Raimond, and S. Haroche, Observing the progressive decoherence of the meter in a quantum measurement, Phys. Rev. Lett. 77, 4887 (1996).

[8] Q. J. Myatt, B. E. King, Q. A. Turchette, C. A. Sackett, D. Kielpinski, W. M. Itano, C. Monroe, and D. J. Wineland, Decoherence of quantum superpositions through coupling to engineered reservoirs, Nature (London) 403, 269 (2000).

[9] A. Einstein, B. Podolsky, and N. Rosen, Can quantum mechanical description of physical reality be considered complete? Phys. Rev. 47, 777 (1935).

[10] R. J. Glauber, Coherent and incoherent states of the radiation field, Phys. Rev. 131, 2766 (1963).

[11] U. M. Titulaer and R. J. Glauber, Density operators for coherent fields, Phys. Rev. 145, 1041 (1966).

[12] S. M. Barnett and P. M. Radmore, Methods in Theoretical Quantum Optics, Oxford University Press, Oxford, 1997.

[13] V. Buzek, H. Moya-Cessa, P. L. Knight, and S. D. L. Phoenix, Schrödinger-cat states in the resonant Jaynes-Cummings model: Collapse and revival of oscillations of the photon-number distribution, Phys. Rev. A 45, 8190 (1992).

[14] M. Brune, S. Haroche, J.-M. Raimond, L. Davidovich, and N. Zagury, Manipulation of photons in a cavity by dispersive atom-field coupling: Quantum non demolition measurements and generation of Schrödinger cat states, Phys. Rev. A 45, 5193 (1992).

[15] W. P. Schleich, Quantum Optics in Phase Space, Wiley, Berlin, 2001.

[16] J.-M. Raimond, T. Meunier, P. Bertet, S. Gleyzes, P. Maioli, A. Auffeves, G. Nogues, M. Brune, and S. Haroche, Probing a quantum field in a photon box, J. Phys. B 38, S535 (2005).

[17] E. P. Wigner, On the quantum correction for thermodynamic equilibrium, Phys. Rev. 40, 749 (1932).

[18] K. E. Cahill and R. J. Glauber, Ordered expansions in boson amplitude operators, Phys. Rev. 177, 1857 (1969).

[19] J. Dalibard, Y. Castin, and K. Mölmer, Wave-function approach to dissipative processes in quantum optics, Phys. Rev. Lett. 68, 580 (1992).

[20] M. B. Plenio and P. L. Knight, The quantum-jump approach to dissipative dynamics in quantum optics, Rev. Mod. Phys. 70, 101 (1998).

[21] T. Sauter, W. Neuhauser, R. Blatt, and P. Toschek, Observation of quantum jumps, Phys. Rev. Lett. 57, 1696 (1986).

[22] J. C. Bergquist, R. G. Hulet, W. M. Itano, and D. J. Wineland, Observation of quantum jumps in a single atom, Phys. Rev. Lett. 57, 1699 (1986).

[23] C. Cohen-Tannoudji and J. Dalibard, Single-atom laser spectroscopy - looking for dark periods in fluorescence, EuroPhys. Lett. 1, 441 (1986).

[24] E. T. Jaynes and F. W. Cummings, Comparison of quantum and semiclassical radiation theories with application to the beam maser, Proc. IEEE 51, 89 (1963). 
[25] S. Haroche and J.-M. Raimond, Manipulation of non-classical field states in a cavity by atom interferometry, In P. Berman, editor, Advances in Atomic and Molecular Physics, supplement 2, page 123. Academic Press, New York, 1994.

[26] S. Haroche. Cavity quantum electrodynamics, In J. Dalibard, J.-M. Raimond, and J. ZinnJustin, editors, Fundamental Systems in Quantum Optics, Les Houches Summer School, Session LIII, page 767. North Holland, Amsterdam, 1992.

[27] P. Maioli, T. Meunier, S. Gleyzes, A. Auffeves, G. Nogues, M. Brune, J.-M. Raimond, and S. Haroche, Non-destructive Rydberg atom counting with mesoscopic fields in a cavity, Phys. Rev. Lett. 94, 113601 (2005).

[28] M. Brune, P. Nussenzveig, F. Schmidt-Kaler, F. Bernardot, A. Maali, J.-M. Raimond, and S. Haroche, From Lamb shifts to light shifts: Vacuum and subphoton cavity fields measured by atomic phase sensitive detection, Phys. Rev. Lett. 72, 3339 (1994).

[29] N. F. Ramsey. Molecular Beams, International series of monographs on physics, Oxford University Press, Oxford, 1985.

[30] P. Bertet, S. Osnaghi, A. Rauschenbeutel, G. Nogues, A. Auffeves, M. Brune, J.-M. Raimond, and S. Haroche, A complementarity experiment with an interferometer at the quantumclassical boundary, Nature (London) 411, 166 (2001).

[31] L. Davidovich, M. Brune, J.-M. Raimond, and S. Haroche, Mesoscopic quantum coherences in cavity QED: Preparation and decoherence monitoring schemes, Phys. Rev. A 53, 1295 (1996).

[32] J.-M. Raimond, M. Brune, and S. Haroche, Reversible decoherence of a mesoscopic superposition of field states, Phys. Rev. Lett. 79, 1964 (1997).

[33] P. Bertet, A. Auffeves, P. Maioli, S. Osnaghi, T. Meunier, M. Brune, J.-M. Raimond, and S. Haroche, Direct measurement of the Wigner function of a one-photon Fock state in a cavity, Phys. Rev. Lett. 89, 200402 (2002).

[34] M. Fortunato, J.-M. Raimond, P. Tombesi, and D. Vitali, Autofeedback scheme for schrödinger cat preservation in microwave cavities, Phys. Rev. A 60, 1687 (1999).

[35] S. Zippilli, D. Vitali, P. Tombesi, and J.-M. Raimond, Scheme for decoherence control in microwave cavities, Phys. Rev. A 67, 052101 (2003).

[36] J. D. Jackson, Classical Electrodynamics, Wiley, New York, second edition, 1975.

[37] A. Faist, E. Geneux, P. Meystre, and A. Quattropani, Coherent radiation in interaction with two-level system, Helv. Phys. Acta 45, 956 (1972).

[38] J. H. Eberly, N. B. Narozhny, and J. J. Sanchez-Mondragon, Periodic spontaneous collapse and revival in a simple quantum model, Phys. Rev. Lett. 44, 1323 (1980).

[39] P. L. Knight and P. M. Radmore, Quantum origin of dephasing and revivals in the coherentstate Jaynes-Cummings model, Phys. Rev. A 26, 676 (1982).

[40] J. Gea-Banacloche, Atom and field evolution in the Jaynes and Cummings model for large initial fields, Phys. Rev. A 44, 5913 (1991).

[41] M. Fleischhauer and W. P. Schleich, Revivals made simple: Poisson summation formula as a key to the revivals in the Jaynes-Cummings model, Phys. Rev. A 47, 4258 (1993).

[42] V. Buzek and P. L. Knight, Quantum interference, superposition states of light and nonclassical effects, In Progress in Optics XXXIV, volume 34, page 1. Elsevier, 1995. 
[43] A. Auffeves, P. Maioli, T. Meunier, S. Gleyzes, G. Nogues, M. Brune, J.-M. Raimond, and S. Haroche, Entanglement of a mesoscopic field with an atom induced by photon graininess in a cavity, Phys. Rev. Lett. 91, 230405 (2003).

[44] G. Morigi, E. Solano, B.-G. Englert, and H. Walther, Measuring irreversible dynamics of a quantum harmonic oscillator, Phys. Rev. A 65, 040102 (2002).

[45] T. Meunier, S. Gleyzes, P. Maioli, A. Auffeves, G. Nogues, M. Brune, J.M. Raimond, and S. Haroche, Rabi oscillations revival induced by time reversal: a test of mesoscopic quantum coherence, Phys. Rev. Lett. 94, 010401 (2005).

[46] L. G. Lutterbach and L. Davidovich, Method for direct measurement of the Wigner function in cavity QED and ion traps, Phys. Rev. Lett. 78, 2547 (1997).

[47] K. Banaszek and K. Wodkiewicz, Testing quantum nonlocality in phase space, Phys. Rev. Lett. 82, 2009 (1999).

[48] A. Aspect, J. Dalibard, and G. Roger, Experimental test of Bell's inequalities using timevarying analysers, Phys. Rev. Lett. 49, 1804 (1982).

[49] A. Zeilinger, Experiment and the foundations of quantum physics, Rev. Mod. Phys. 71, S288 (1999).

[50] P. Milman, A. Auffeves, F. Yamaguchi, M. Brune, J.M. Raimond, and S. Haroche, A proposal to test Bell's inequalities with mesoscopic non-local states in cavity qed, Eur. Phys. J. D 32, $233(2005)$. 